\title{
Classical or Quantum? What is Reality?
}

\author{
J. J. Sławianowski and V. Kovalchuk \\ Additional information is available at the end of the chapter
}

http://dx.doi.org/10.5772/59115

\section{Introduction}

Discussion of those questions and the struggle with the well-known paradoxes of quantum mechanics like decoherence and measurement problems is as old as the quantum mechanics itself. This was just the reason for the search of formulations of any of both theories in terms of concepts characteristic for the other one. It would be injustice to condemn or disqualify those attempts. They were fruitful, enabled one to solve many concrete problems and did shed some light onto the mutual relationship of both theories. Nevertheless all mysterious features of quantum theory remained mysterious as they were. There is a whole spectrum of views; let us quote the dominant ones in a very simplified form:

1. In principle everything is "quantum". The "classical" is an approximation of large quantum numbers in large, macroscopic subsystems of the Universe. In a sense it is a kind of "illusion" well working in a restricted range of physical phenomena.

2. Finally everything should be "classical" and the "quantum" model is phenomenological and temporary, in any case incomplete. Very often one formulates the conjecture that it is the linearity of quantum theory that is guilty. In any case: what other physical factor might be responsible for the discrepancy between unitary evolution of the unobserved microsystem and the measurement reduction phenomena?

3. Physical reality is dualistically built of two incompatible elements: quantum and classical. They are joined into a single whole via statistical interpretation of the wave function. This unification is rather mysterious from the purely classical point of view, nevertheless rigorously described mathematically by the standard probabilistic interpretation. This view is relatively popular, although it is not free of the solliptic or even divine ideas. Let us mention that both those ideas are physically justified.

There are many papers concerning the first and third possibility. But the second item, i.e., nonlinearity, is not very popular, although it seems to be a good candidate for explaining quantum paradoxes and exorcising the solliptic or any dualistic ideas. Incidentally, from the point of view of the development of physics and other natural sciences this reluctance 
of physicists to nonlinearity in quantum mechanics is rather strange. It contradicts the whole history of physics. Usually one begins, when it is only possible, from linear models, but later on one introduces nonlinear terms to equations. And now we witness the unbelievable development of nonlinear methods, also essentially nonlinear ones, when there is no well-defined linear background term perturbed by a nonlinear correction. It is simply fashionable to formulate and discuss models based on the non-perturbative nonlinearity of geometric origin. Why really to stick to linearity in so fundamental science as quantum physics? Let us also mention that there are objects in the nano-scale like graphenes, fullerenes, and very large molecules the behaviour of which is placed somewhere in the convolution region of the quantum and classical theory. Let us also remind that there are nonlinear computational methods like the Fermi-Dirac procedure in the usual quantum mechanics. It is not excluded that some more fundamental nonlinearity may be formulated and efficiently used.

One of reasons of the reluctance of physicists to nonlinearity in quanta is some non-physical arbitrariness of the nonlinear dynamical models. And really, there were various models introduced "by hand". But recently some essentially nonlinear, non-perturbative models based on deep geometric ideas were suggested. For example, we mean here papers by Doebner and Goldin with co-workers [4, 5], works by Svetlichny [17], and some of our publications [13].

Below we consider two methods of quasi-classical analysis: one based on the limit transition with the Planck constant tending to zero and one based on the analysis of quickly varying wave functions with many modes. They are both connected with the mechanical-optical analogy and both are useful. Namely, the theoretical approach to physics is one based on differential equations, usually partial ones in appropriate variables. But they also contain plenty of so-called physical constants usually experimentally fixed. Let us mention, e.g., $\hbar$, $c, G, e, k$, etc. (respectively, the Planck constant, velocity of light in vacuum, gravitational constant, elementary electric charge, Boltzmann constant, etc.). The system of natural units like $\hbar=1, c=1$ is not appropriate. First of all because it is incomparable with the limit transition to zero or infinity. The only solution of the problem is to introduce $\hbar, c$, etc. as "controlling" parameters and to follow quietly (as possible) the consequence of the mentioned manipulations. Let us mention that there are two aspects of the controlling status of those constants. First, one can think about the anthropic principle and perhaps the divine origin of them. The second aspect may be more material. One can, e.g., think on them as fields and even to formulate the corresponding field or motion equations. Let us mention, incidentally, that putting $\hbar=0$ we get rid of all paradoxes of quantum mechanics, but obtain the non-realistic world with infinities in electromagnetic radiation theory.

In a sense it might seem strange that quantum mechanics was not formulated or at least suggested some hundred years earlier. Though both geometric and physical optics seemed to be known in the deep of XIX century. And one was also aware that the geometric optics is a short-wave asymptotics of the wave theory. And the analogy between mechanics and geometric optics was also known. Mathematically it was based on the similarity of the Hamilton-Jacobi and eikonal equations. But one interpreted it as a formal similarity between description of single particle trajectories and the geometric theory of optical waves moving along the eikonal rays. The mechanical "waves" were not interpreted as the dynamics of a real wave process even in the sense of the Schrödinger wave picture (as a matter of fact also incorrect, as it turned out later on). It seems that the deciding circumstance here was the absence of the Planck constant, the physical quantity of the dimension of 
action. It is necessary to replace the Hamilton-Jacobi action $S\left(t, q^{i}\right)$ by the wave containing factor $\exp (i / \hbar) S(q)$ with the dimensionless argument. It is strange that the $\hbar$-divisor was discovered by Planck in phenomena of electromagnetic radiation, where it is deeply hidden from the direct observation. And only when it was discovered by Planck, the further procedure was open by Planck himself, Bohr, Sommerfeld, de Broglie, Heisenberg, Schrödinger, and many others.

\section{Weyl-Wigner-Moyal-Ville description}

It is neither very easy nor automatic to perform the limit transition $\hbar \rightarrow 0$ in quantum-mechanical equations. In any case passing to zero with $\hbar$ in quantum-mechanical wave functions leads to meaningless results. Rather, one should separately use the explicit $\hbar$-dependence of the modulus and phase of the wave function

$$
\Psi=\sqrt{D} \exp \left(\frac{i}{\hbar} S\right)=f \exp \left(\frac{i}{\hbar} S\right)
$$

and substitute this to the Schrödinger equation (or any other wave equation). And then one should write the system of equations resulting from the comparison of coefficients at the same (but theoretically all possible) powers of $\hbar$. It is important to remember that $D, S$ are power series of $\hbar$, but the divisor $\hbar$ under the exp sign is universally present in equations. The simplest, heuristic way is to use the Weyl-Wigner-Moyal-Ville (WWMV) star product of operators when the phase space is $\mathbb{R}^{2 n}$ (or any $2 n$-dimensional linear space).

Let $\left(q^{1}, \ldots, q^{n} ; p_{1}, \ldots, p_{n}\right)$ be affine phase space coordinates, $\left(q^{1}, \ldots, q^{n}\right)$ - the underlying configuration space variables, and $\left(p_{1}, \ldots, p_{n}\right)$ - the induced momentum variables. The operators acting (in principle) in $L^{2}\left(q^{1}, \ldots, q^{n}\right)$ are represented by their kernels-functions or rather distributions when we do not insist on remaining within $L^{2}(Q)$ :

$$
(\mathbf{A} \Psi)(\bar{q})=\int A\left[\bar{q}, \bar{q}^{\prime}\right] \Psi\left(\bar{q}^{\prime}\right) d_{n} \bar{q}^{\prime}
$$

What concerns the adjective "distribution-like" let us stress that such important operators as identity, position, and linear momentum are just represented by distributions:

$$
\mathbf{1}\left[\bar{q}, \bar{q}^{\prime}\right]=\delta\left(\bar{q}-\bar{q}^{\prime}\right), \mathbf{Q}^{a}\left[\bar{q}, \bar{q}^{\prime}\right]=q^{a} \delta\left(\bar{q}-\bar{q}^{\prime}\right), \mathbf{P}_{a}\left[\bar{q}, \bar{q}^{\prime}\right]=\frac{\hbar}{i} \frac{\partial}{\partial q^{a}} \delta\left(\bar{q}-\bar{q}^{\prime}\right)
$$

According to the Weyl-Wigner-Moyal-Ville prescription one can represent any function $A\left(q^{1}, \ldots, q^{n} ; p_{1}, \ldots, p_{n}\right)$ by the kernel

$$
A\left[\bar{q}, \bar{q}^{\prime}\right]=\int A\left(\frac{1}{2}\left(\bar{q}+\bar{q}^{\prime}\right), \bar{p}\right) \exp \left(\frac{i}{\hbar} \bar{p} \cdot\left(\bar{q}-\bar{q}^{\prime}\right)\right) \frac{d_{n} \bar{p}}{(2 \pi \hbar)^{n}}
$$


and conversely, inverting the Fourier transform:

$$
A(\bar{q}, \bar{p})=\int \exp \left(-\frac{i}{\hbar} \bar{p} \cdot \bar{\alpha}\right) A\left[\bar{q}+\frac{\bar{\alpha}}{2}, \bar{q}-\frac{\bar{\alpha}}{2}\right] d_{n} \bar{\alpha} .
$$

Let us remind that this is a consequence of the Weyl-Wigner-Moyal-Ville star product of the pair of phase-space functions on the affine phase space:

$$
\begin{aligned}
(A * B)(z) & =2^{2 n} \int \exp \left(\frac{2 i}{\hbar} \Gamma\left(z-z_{1}, z-z_{2}\right)\right) A\left(z_{1}\right) B\left(z_{2}\right) d \mu\left(z_{1}\right) d \mu\left(z_{2}\right), \\
d \mu(z) & =d \mu(\bar{q}, \bar{p})=\frac{1}{(2 \pi \hbar)^{n}} d q^{1} \ldots d q^{n} d p_{1} \ldots d p_{n}
\end{aligned}
$$

where in the $(\bar{q}, \bar{p})$-basis $\Gamma=\left[\begin{array}{cc}O & -I \\ I & O\end{array}\right], I$ is the $n \times n$ identity matrix, and $O$ is the $n \times n$ matrix composed of zeros. Obviously, the product $A * B$ is isomorphic to the product of operators represented by the "matrix" rule:

$$
[A B]\left(\bar{q}, \bar{q}^{\prime}\right)=\int A\left[\bar{q}, \bar{q}^{\prime \prime}\right] B\left[\bar{q}^{\prime \prime}, \bar{q}^{\prime}\right] d_{n} q^{\prime \prime}
$$

And Hermitian conjugate of operators is represented by the complex conjugate of phase-space functions. The above composition of phase-space functions is non-local and in general the positively definite operators (like, e.g., density operators $\rho$ ) are not represented by non-negative phase-space functions.

It is clear from the above formulae that the action of the operator $\mathbf{A}$ on the configuration space function $\Psi$ is given by

$$
(\mathbf{A} \Psi)(\bar{q})=\frac{1}{(2 \pi \hbar)^{n}} \int \exp \left(\frac{i}{\hbar} \bar{p} \cdot\left(\bar{q}-\bar{q}^{\prime}\right)\right) A\left(\frac{1}{2}\left(\bar{q}+\bar{q}^{\prime}\right), \bar{p}\right) \Psi\left(\bar{q}^{\prime}\right) d_{n} \bar{q}^{\prime} d_{n} \bar{p}
$$

Let us remind a few properties of the Weyl-Wigner-Moyal-Ville product of the phase-space functions. So, it is bilinear and associative and preserves the complex conjugation:

$$
\begin{aligned}
& (\lambda A+\mu B) * C=\lambda A * C+\mu B * C, \quad(A * B) * C=A *(B * C), \\
& C *(\lambda A+\mu B)=\lambda C * A+\mu C * B, \overline{A * B}=\bar{B} * \bar{A},
\end{aligned}
$$

where $\mathbf{A}^{+}$is represented by $\bar{A}$. Besides $1 * A=A * 1, \bar{A} * A \neq 0$, if $A \neq 0$ a.e., $\int A * B d \mu=$ $\int A B d \mu$, but in general $\int A * B * C d \mu \neq \int A B C d \mu$. Let us notice that

$$
\operatorname{Tr} \mathbf{A}=\int A(\bar{z}) d \mu(\bar{z}), \quad\langle\mathbf{A}, \mathbf{B}\rangle=\operatorname{Tr}\left(\mathbf{A}^{+} \mathbf{B}\right)=\int \overline{A(\bar{z})} B(\bar{z}) d \mu(\bar{z}),
$$


and $\langle C * A, B\rangle=\langle A, \bar{C} * B\rangle=\langle C, B * \bar{A}\rangle$. Obviously, the star-product of phase-space functions is non-commutative, just as the operator product. Let us quote some formulae concerning non-commutativity and commutativity:

$$
\begin{gathered}
q^{a} * p_{b}=q^{a} p_{b}+\frac{i \hbar}{2} \delta^{a}{ }_{b}, \quad p_{b} * q^{a}=p_{b} q^{a}-\frac{i \hbar}{2} \delta^{a}{ }_{b}, \\
q^{a} * A(q, p)=q^{a} A(q, p)+\frac{i \hbar}{2} \frac{\partial A}{\partial p_{a}}, A(q, p) * q^{a}=A(q, p) q^{a}-\frac{i \hbar}{2} \frac{\partial A}{\partial p_{a}}, \\
p_{a} * A(q, p)=p_{a} A(q, p)-\frac{i \hbar}{2} \frac{\partial A}{\partial q^{a}}, A(q, p) * p_{a}=A(q, p) p_{a}+\frac{i \hbar}{2} \frac{\partial A}{\partial q^{a}},
\end{gathered}
$$

and obviously for functions depending only on one kind of variables

$$
\begin{aligned}
& (A * B)(q)=A(q) * B(q)=A(q) B(q)=(A B)(q), \\
& (A * B)(p)=A(p) * B(p)=A(p) B(p)=(A B)(p) .
\end{aligned}
$$

The star-product is evidently invariant under the action of affine symplectic group, $(U(A, t) f) *(U(A, t) g)=U(A, t)(f * g)$, where $t$ is a translation vector in $\mathbb{R}^{2 n}$ and $A$ is a linear symplectic transformation, $\Gamma_{k l} A^{k}{ }_{a} A_{b}^{l}=\Gamma_{a b},(U(A, t) f)(z)=f(A z+t)$.

Quantum states are described by density operators $\rho$ which are Hermitian, normalized to unity and positive: $\left\langle\boldsymbol{\rho} \mid \mathbf{A}^{+} \mathbf{A}\right\rangle=\operatorname{Tr}\left(\boldsymbol{\rho} \mathbf{A}^{+} \mathbf{A}\right) \geq 0, \operatorname{Tr} \boldsymbol{\rho}=1$. To be honest, one can also live without the last normalization condition. When the condition is satisfied, we have $\operatorname{Tr} \rho^{2} \leq$ $\operatorname{Tr} \boldsymbol{\rho}=1$. Particularly important are pure states described by projectors, $\widehat{\boldsymbol{\rho}}^{2}=\widehat{\boldsymbol{\rho}}, \widehat{\boldsymbol{\rho}}=|\Psi\rangle\langle\Psi|$, $\rho\left[\bar{q}, \bar{q}^{\prime}\right]=\Psi(\bar{q}) \bar{\Psi}\left(\bar{q}^{\prime}\right)$. They are related to the corresponding wave function $\Psi$ as follows:

$$
\rho\left(\bar{q}, \bar{q}^{\prime}\right)=\frac{1}{(2 \pi)^{n}} \int \bar{\Psi}\left(\bar{q}-\frac{\hbar}{2} \bar{\tau}\right) \exp (-i \bar{\tau} \cdot \bar{p}) \Psi\left(q+\frac{\hbar}{2} \bar{\tau}\right) d_{n} \bar{\tau}
$$

In general it takes on negative values, nevertheless it is positive in the quantum-mechanical sense:

$$
\langle\rho \mid \bar{B} * B\rangle=\int \rho(z)(\bar{B} * B)(z) d \mu(z)>0
$$

for all functions $B$. The exceptional Wigner functions are positive in the literal sense and are exponential:

$$
E_{(\bar{\xi}, \bar{\pi})}(\bar{q}, \bar{p})=\frac{1}{(\pi \hbar)^{n}} \exp \left(-\frac{1}{\hbar}\left((\bar{q}-\bar{\xi})^{2}+(\bar{p}-\bar{\pi})^{2}\right)\right) .
$$

It is clear that they represent pure states,

$$
E_{(\bar{\xi}, \bar{\pi})} * E_{(\bar{\xi}, \bar{\pi})}=E_{(\bar{\xi}, \bar{\pi})^{\prime}} \quad \int E_{(\bar{\xi}, \bar{\pi})}(\bar{q}, \bar{p}) d_{n} \bar{q} \frac{d_{n} \bar{p}}{(2 \pi \hbar)^{n}}=1 .
$$


They are coherent states strongly concentrated in the phase space about the point $(\bar{\xi}, \bar{\pi})$. Therefore, the coarse-grained quantity, so-called Husimi distribution,

$$
\widetilde{\rho}(\bar{q}, \bar{p})=\int E_{(\bar{q}, \bar{p})}(\bar{\xi}, \bar{\pi}) \rho(\bar{\xi}, \bar{\pi}) d_{n} \bar{\xi} \frac{d_{n} \bar{\pi}}{(2 \pi \hbar)^{n}}
$$

admits an approximate interpretation of the literally positively-definite probability distribution obtained from the Wigner function $\rho$. Indeed, $E_{(\bar{q}, \bar{p})}$ is in a sense a pure state Wigner function concentrated at $(\bar{q}, \bar{p})$ and therefore the above integral is a probability density for the system to be found in the phase-space cell at $(\bar{q}, \bar{p})$ when it is known to be in a Wigner state $\rho$. This interpretation is not bad and certainly $\widetilde{\rho}$ is something that in a sense gives an account of the probability distribution to be found in an $\hbar^{n}$-volume cell about every $(\bar{q}, \bar{p})$. There is only one drawback of this interpretation. Namely, unlike the true Weyl-Wigner-Moyal-Ville distributions, literally non-positive, the Husimi distributions (22) have non-satisfactory, bad marginal properties, because

$$
\int \widetilde{\rho}(\bar{q}, \bar{p}) d_{n} \bar{q} \neq \bar{\Psi}(\bar{p}) \widehat{\Psi}(\bar{p}), \quad \int \widetilde{\rho}(\bar{q}, \bar{p}) \frac{d_{n} \bar{p}}{(2 \pi \hbar)^{n}} \neq \bar{\Psi}(\bar{q}) \Psi(\bar{q}) .
$$

Here, obviously, $\Psi, \widehat{\Psi}$ are the wave functions underlying $\rho$, respectively in the coordinate and momentum representations. For the $\rho$ itself the above inequalities become exact equalities.

Let us now discuss the problem of the WKB approximation from the point of view of the above remarks. It is clear that from the point of view of the above statements, in the lowest-order approximation of $D, S$ in $\hbar$, we have the following $\hbar$-independent interpretation of the $D, S$-functions in terms of the expectation values:

$$
\begin{aligned}
\left\langle\Psi\left|\mathbf{Q}^{i}\right| \Psi\right\rangle & =\int D\left(q^{1}, \ldots, q^{n}\right) q^{i} d q^{1} \ldots d q^{n} \\
\left\langle\Psi\left|\mathbf{P}_{i}\right| \Psi\right\rangle & =\int D\left(q^{1}, \ldots, q^{n}\right) \partial_{i} S\left(q^{1}, \ldots, q^{n}\right) d q^{1} \ldots d q^{n}
\end{aligned}
$$

It is clear that the Planck constant $\hbar$ is absent in those expressions, so really the functions $D, S$ are $\hbar$-independent up to higher orders. In any case it is so at places distant from the turning points. Let us consider the $n$-dimensional submanifold given by equations $p_{i}=\partial S / \partial q^{i}$, $i=1, \ldots, n$, i.e., $F_{i}=p_{i}-\left(\partial S / \partial q^{i}\right)=0$. This submanifold, $\mathfrak{m}_{S}$, is a special case of what is called Lagrangian manifold, because the Poisson brackets of the left-hand sides of its equations vanish; moreover, they vanish after the restriction to $\mathfrak{m}_{S}$,

$$
\left\{F_{i}, F_{j}\right\}=\frac{\partial F_{i}}{\partial q^{a}} \frac{\partial F_{j}}{\partial p_{a}}-\frac{\partial F_{i}}{\partial p_{a}} \frac{\partial F_{j}}{\partial q^{a}}=\left(\partial_{i j}^{2}-\partial_{j i}^{2}\right) S=0
$$

Let us take the singular probability distribution concentrated on $\mathfrak{m}_{S}$ : 


$$
\begin{aligned}
\rho_{\mathrm{cl}}[D, S] & =\lim _{\hbar \rightarrow 0} \rho[D, S]=D\left(q^{1}, \ldots, q^{n}\right) \delta\left(p_{1}-\frac{\partial S}{\partial q^{1}}\right) \ldots \delta\left(p_{n}-\frac{\partial S}{\partial q^{n}}\right) \\
& =\left|\Psi\left(q^{1}, \ldots, q^{n}\right)\right|^{2} \delta\left(p_{1}-\frac{\partial S}{\partial q^{1}}\right) \ldots \delta\left(p_{n}-\frac{\partial S}{\partial q^{n}}\right) .
\end{aligned}
$$

Obviously, it is different from $\rho(q, p)$, nevertheless the expectation values of $q^{i}, p_{j}$ and their linear combinations on $\rho_{\mathrm{cl}}$ are just the same as those on $\rho$,

$$
\begin{aligned}
\left\langle\Psi\left|\alpha_{i} \mathbf{q}^{i}+\beta^{i} \mathbf{p}_{i}\right| \Psi\right\rangle & =\int\left(\alpha_{i} q^{i}+\beta^{i} p_{i}\right) \rho[D, S] d_{n} \bar{q} \frac{d_{n} \bar{p}}{(2 \pi \hbar)^{n}} \\
& =\int\left(\alpha_{i} q^{i}+\beta^{i} p_{i}\right) \rho_{\mathrm{cl}}[D, S] d_{n} \bar{q} \frac{d_{n} \bar{p}}{(2 \pi \hbar)^{n}}
\end{aligned}
$$

Let us mention that all limit transitions here, in particular the one between $\rho[D, S]$ and $\rho_{\mathrm{cl}}[D, S]$ are meant in the distribution theory sense.

It is important that the Weyl-Wigner-Moyal-Ville product may be expanded as a power series in $\hbar$ and that the functional coefficients are interpretable in terms of the symplectic geometry of the classical phase space. The first two terms of the expansion are given by

$$
A * B \simeq A B+\frac{i \hbar}{2}\{A, B\}+\ldots,
$$

the next terms are given by the multiple Poisson brackets. In any case, the Weyl-Wigner-Moyal-Ville product and the corresponding quantum Poisson bracket are given in the limit $\hbar \rightarrow 0$ by the following $\hbar$-independent expressions:

$$
\begin{aligned}
\lim _{\hbar \rightarrow 0} A * B & =A B, \\
\lim _{\hbar \rightarrow 0}\{A, B\}_{\mathrm{QPB}}=\lim _{\hbar \rightarrow 0} \frac{1}{i \hbar}(A * B-B * A) & =\{A, B\} .
\end{aligned}
$$

Let us mention that these formulae have interesting features and interpretation. Namely, the eigenequation for the wave function implies the following eigenequation for the corresponding density operator: $\mathbf{A} \boldsymbol{\rho}=a \boldsymbol{\rho}$, i.e., in terms of the Weyl-Wigner-Moyal-Ville approach $A * \rho=a \rho$. But this implies $\rho * A=a \rho$ if $A$ is real, i.e., $\mathbf{A}$ is hermitian, and therefore

$$
[\mathbf{A}, \boldsymbol{\rho}]_{\mathrm{QPB}}=\frac{1}{i \hbar}[\mathbf{A}, \boldsymbol{\rho}]=0, \quad \text { thus, } \quad \frac{1}{i \hbar}(A * \rho-\rho * A)=0 .
$$


On the quantum level this equation is a direct consequence of the eigenequation $A * \rho=a \rho$. But these equations have quite different qualitative interpretation in physical terms. Namely, $\mathbf{A} \boldsymbol{\rho}=a \boldsymbol{\rho}$ has a purely informational content. It tells us that on the state $\boldsymbol{\rho}$, or $\rho$ in the Weyl-Wigner-Moyal-Ville language, the physical quantity $\mathbf{A}(A$ in the Weyl-Wigner-Moyal-Ville terms) takes spread-freely the value $a$. This is the purely informational property. But the Poisson bracket property, mathematically following from it, has a qualitatively different interpretation, namely, such a $\rho$ is invariant under the one-parameter group of unitary transformations generated by $\mathbf{A}(A)$,

$$
\exp \left(\frac{i}{\hbar} \mathbf{A} \tau\right) \boldsymbol{\rho} \exp \left(-\frac{i}{\hbar} \mathbf{A} \tau\right)=\boldsymbol{\rho}
$$

This is a symmetry property. Therefore, on the quantum level information implies symmetry. But in classical physics Poisson bracket and the pointwise product of functions are algebraically independent. Therefore, information and symmetry of statistical states become logically independent. This implies that in the classical limit Schrödinger equation or the corresponding eigenequation for the density operator must be in the lowest order of approximation replaced by the pair of equations for the phase and modulus of the wave function. Therefore, substituting (1) to (9) and taking the limit $\hbar \rightarrow 0$ we obtain:

$$
(\mathbf{A} \Psi)(q) \approx A\left(q^{i}, \frac{\partial S}{\partial q^{i}}\right) \Psi(q)+\frac{\hbar}{i}\left(£_{v} f\right) \exp \left(\frac{i}{\hbar} S(q)\right)
$$

higher order terms in $\hbar$ are omitted. The symbol $£_{v}$ denotes the Lie derivative of $f$ with respect to the vector field $v[A, S]$ which equals

$$
v^{i}=\frac{\partial A}{\partial p_{i}}\left(q^{j}, \frac{\partial S}{\partial q^{j}}\right)
$$

In spite of the use of analytical symbols, $v^{i}$ is a well-defined vector field tangent to the manifold $\mathfrak{m}_{S}$ given by equations $p_{j}=\partial S / \partial q^{j}, j=1, \ldots, n$. It is obtained from the Hamiltonian vector field generated by the function $A$,

$$
X[A]=\frac{\partial A}{\partial p_{i}} \frac{\partial}{\partial q^{i}}-\frac{\partial A}{\partial q^{i}} \frac{\partial}{\partial p_{i}}
$$

This vector field is tangent to $\mathfrak{m}_{S}$, so we restrict it to some vector field on this manifold and project it to the configuration space $Q$, i.e., to the manifold of $q^{a}$-variables. It is clear that $f$ geometrically is not a scalar field, but the scalar $W$-density of weight $1 / 2$. Therefore,

$$
£_{v} f=v^{a} \frac{\partial f}{\partial q^{a}}+\frac{1}{2} \frac{\partial v^{a}}{\partial q^{a}} f
$$


$D$ is a scalar density of weight one, thus,

$$
£_{v} D=v^{a} \frac{\partial D}{\partial q^{a}}+\frac{\partial v^{a}}{\partial q^{a}} D=\frac{\partial}{\partial q^{a}}\left(D v^{a}\right) .
$$

If we consider the Schrödinger equation

$$
i \hbar \frac{\partial \Psi}{\partial t}=\mathbf{H} \Psi
$$

then in the quasiclassical limit we obtain the following system of equations:

$$
\frac{\partial S}{\partial t}+H\left(q, \frac{\partial S}{\partial q}, t\right)=0, \quad \frac{\partial D}{\partial t}+\frac{\partial}{\partial q^{a}}\left(D \frac{\partial H}{\partial p_{a}}\left(q, \frac{\partial S}{\partial q}\right)\right)=0
$$

This is the system composed of the Hamilton-Jacobi equation for $S$ and the continuity equation for $D$. The second equation is dependent on the solution of the first one. Geometrically it may be written in the form

$$
\frac{\partial D}{\partial t}+£_{v[H, S]} D=0
$$

Let us take a system of $n$ functions $A_{i}$ on the phase space with pairwise vanishing Weyl-Wigner-Moyal-Ville commutators,

$$
A_{i} * A_{j}-A_{j} * A_{i}=0
$$

Consider the family of eigenequations for the Weyl-Wigner-Moyal-Ville density function $\rho$ :

$$
A_{i} * \rho=a_{i} \rho
$$

Obviously, they imply that $(1 / i \hbar)\left(A_{i} * \rho-\rho * A_{i}\right)=0$. In the classical limit this system becomes

$$
A_{i} \rho=a_{i} \rho, \quad\left\{A_{i}, \rho\right\}=0 .
$$

The quantum compatibility condition (42) for (43) implies that in the classical limit the corresponding condition for (44), i.e., $\left\{A_{i}, A_{j}\right\}=0$, also holds. The corresponding solution for (44) may be given as:

$$
\rho(q, p)=\delta\left(A_{1}(q, p)-a_{1}\right) \ldots \delta\left(A_{n}(q, p)-a_{n}\right) .
$$

To be more precise, this holds when $A_{1}, \ldots, A_{n}$ is a system of functionally independent analytic functions. This distribution is concentrated on the Lagrangian manifold $\mathfrak{m}_{(A, a)}$ given 
by equations: $A_{i}(q, p)=a_{i}, i=1, \ldots, n$. Solving them with respect to $p_{i}$ we obtain the transformed equations in the potential form: $p_{j}=\partial S(q, a) / \partial q^{j}$. Short calculation shows that $\rho$ may be written as follows:

$$
\rho(q, p)=\left|\operatorname{det}\left[\frac{\partial^{2} S}{\partial q^{i} \partial a^{j}}\right]\right| \delta\left(p_{1}-\frac{\partial S(q, a)}{\partial q^{1}}\right) \ldots \delta\left(p_{n}-\frac{\partial S(q, a)}{\partial q^{n}}\right) .
$$

The quantity $\operatorname{det}\left[\partial^{2} S / \partial q^{i} \partial a^{j}\right]$ is known as the Van Vleck determinant [20]. The corresponding quasiclassical wave function is given by:

$$
\Psi(q, a)=\sqrt{\operatorname{det}\left[\frac{\partial^{2} S}{\partial q^{i} \partial a^{j}}\right]} \exp \left(\frac{i}{\hbar} S(q, a)\right) .
$$

This expression is convenient when one of the functions $A_{1}, \ldots, A_{n}$ is physically interpretable as a Hamiltonian $H$. Or when Hamiltonian is a simple function of other quantities $A_{i}-$ constants of motion, $H=E\left(A_{1}(q, p), \ldots, A_{n}(q, p)\right)$. Then the function

$$
\Psi(q, t ; a)=\sqrt{\operatorname{det}\left[\frac{\partial^{2} S}{\partial q^{i} \partial a^{j}}\right]} \exp \left(\frac{i}{\hbar} S(q, a)-E\left(a_{1}, \ldots, a_{n}, t\right)\right) .
$$

is an approximate quasiclassical solution of the Schrödinger equation (39) with the continuous spectrum of $A_{1}, \ldots, A_{n}$. And here some additional remarks are necessary. The first one is that (47), (48) are valid only far from the turning points. So, they are valid only in the non-compact spaces $\mathbb{R}^{n}, \mathbb{R}^{2 n}$, when there is no quantization of $A_{j}$ at all, or one must modify them so as to admit compact configuration spaces. But then the above version of the Weyl-Wigner-Moyal-Ville formalism does not work and must be replaced by something else. Some way to remain within the framework is to unify the solutions (48) with the quantization of $A_{1}, \ldots, A_{n}$ by the Bohr-Sommerfeld quantum conditions. Roughly speaking, the idea is then that only such submanifolds $\mathfrak{m}_{\left(a^{1}, \ldots, a^{n}\right)}$ are admitted that the periods of $\omega=p_{i} d q^{i}$ on $\mathfrak{m}_{\left(a^{1}, \ldots, a^{n}\right)}$ are integer multiples of the Planck constant. This condition gives rise to the "quantization" of $A_{1}, \ldots, A_{n}$. And this is what one really does in the Old Quantum Theory. But in general some difficulties appear on the level of wave functions (47), (48), namely one has to use some Maslov modifications and use the Airy special functions.

Nevertheless, the very heart of idea survives: quasiclassical pure quantum states are represented by probability distributions concentrated on $n$-dimensional submanifolds of the phase space; let us repeat that $n$ is the number of degrees of freedom. At least locally the expressions (47), (48) are qualitatively correct. This is very interesting from the geometrical point of view. Namely, in spite of using analytic expressions, the Van Vleck determinant is a well-defined, coordinate-independent scalar density of weight two both in the configuration space $Q$ ( $q^{a}$-variables) and in the $\mathbb{R}^{n}$-space of the values $a^{1}, \ldots, a^{n}$ of constants of motion $A_{1}, \ldots, A_{n}$. And its square root is a well-defined scalar $W$-density of weight one. By its very geometric interpretation, this quantity is a priori the best candidate for the quasiclassical probability distribution of the wave functions (47), (48). Obviously, the care must be taken 
concerning the mentioned problems, in particular the behaviour at the classical turning points.

In any case, expressions (47), (48) are almost true (in the quasiclassical sense), when the variables $q^{i}$ are taken modulo $2 \pi$, i.e., when the configuration space is topologically a torus, and when there are no turning points at all. Then the Bohr-Sommerfeld quantum conditions work literally (in approximation) and there is no need to introduce the Airy functions. And Van Vleck determinant is a good approximation to the quantum density function.

Summary of Section 2: The main message following from the above study is that the classical limit transition, when correctly carried out, indicates that it is not points of the classical phase space, but rather $n$-parameter Lagrangian submanifolds in the phase space that is to correspond to the quantum pure states ( $n$ is the number of degrees of freedom). Or more precisely, it is probability distributions on those manifolds that are to describe the pure states. When the time variable is taken into account, then it turns out that the pure states evolutions are what J. L. Synge used to call the coherent $n$-parameter families of classical trajectories. It is in a sense a surprising result that both on the level of wave functions phases and on the level of probability distributions, the corresponding quantities may be a priori guessed on the basis of the classical Hamilton-Jacobi theory and the Van Vleck determinant following from it.

\section{Symplectic and contact interpretation}

Our arguments above were based on the assumed affine geometry of the phase space. However, it is clear that this fact is not very important. It did not influence our views. Affine geometry and the Weyl-Wigner-Moyal-Ville procedure were merely the auxiliary tools of our analysis. Nevertheless, it is convenient to comment our results in general symplectic terms.

Let $(P, \gamma)$ be a symplectic manifold, i.e., a differential manifold $P$ endowed with the differential two-form $\gamma$ satisfying the following conditions: it is closed and non-degenerate. In coordinates $\xi^{a}$ this means that

$$
\gamma=\frac{1}{2} \gamma_{a b} d \xi^{a} \wedge d \xi^{b}
$$

where $\gamma_{a b, c}+\gamma_{b c, a}+\gamma_{c a, b}=0$, $\operatorname{det}\left[\gamma_{a b}\right] \neq 0$ all over $P$. The comma symbol denotes the partial derivative. Therefore, $\operatorname{dim} P=2 n, n$ being natural. As $d \gamma=0$, then locally $\gamma=d \omega$. Not always, but in majority of applications $P$ is a cotangent bundle over some $n$-dimensional configuration space,

$$
P=T^{*} Q=\bigcup_{q \in Q} T_{q}^{*} Q
$$

where $T_{q} Q, T_{q}^{*} Q$ denote as usual the tangent space at $q \in Q$ and its dual - the cotangent space. If $q^{i}, i=1, \ldots, n$, are coordinates in an open domain of $Q$, then the induced coordinates in $T^{*} Q$ are denoted by $\left(q^{i}, p_{i}\right)$, where $p_{i}$ are components of the canonical 
momentum attached of $q \in Q$. This structure gives rise to the Cartan one-form $\omega$ given locally by $\omega=p_{i} d q^{i}$; the coordinate-free definition is easily possible but we do not quote it here. In any case the symplectic form in $T^{*} Q$ is given by $\gamma=d \omega=d p_{i} \wedge d q^{i}$. Being non-degenerate, $\gamma$ does possess the inverse form $\widetilde{\gamma}$ with coordinates $\gamma^{a b}$ such that $\gamma^{a c} \gamma_{c b}=\delta^{a}{ }_{b}$. This gives rise to the Poisson bracket construction

$$
\{F, G\}=\gamma^{a b} \frac{\partial F}{\partial \xi^{a}} \frac{\partial G}{\partial \xi^{b}},
$$

in the induced coordinates $\left(q^{i}, p_{i}\right)$ :

$$
\{F, G\}=\frac{\partial F}{\partial q^{a}} \frac{\partial G}{\partial p_{a}}-\frac{\partial F}{\partial p_{a}} \frac{\partial G}{\partial q^{a}} .
$$

Canonical transformations preserve the two-form $\gamma, \varphi_{*} \gamma=\gamma$, and infinitesimal ones, i.e., canonical vector fields $X$ satisfy $£_{X} \gamma=0$. Of course, the identity $\left.\left.£_{X} \gamma=(X\rfloor d \gamma\right)+d(X\rfloor \gamma\right)$ implies that because of $d \gamma=0$,

$$
(d(X\rfloor \gamma))_{a b}=\left(X^{c} \gamma_{c a}\right)_{, b}-\left(X^{c} \gamma_{c b}\right)_{, a}=0
$$

therefore, at least locally the vector field $X$ is Hamiltonian $(X\rfloor \gamma)_{a}=X^{c} \gamma_{c a}=-\partial F / \partial \xi^{a}$. It is denoted by $X_{F}=-\widetilde{d F}$ and called the Hamiltonian vector field generated by the local Hamiltonian $F$. If $F$ is globally one-valued, we say that $X_{F}$ is a Hamiltonian field generated by $F$. Therefore, unlike the symmetry group of the symmetric metric tensor on a manifold $M$, which is a finite-dimensional Lie group of dimension at most $n(n+1) / 2$, the group of symplectomorphisms, i.e., one of canonical transformations is always infinite-dimensional, labelled by arbitrary sufficiently smooth functions on $P$.

An important problem is a classification of submanifolds in a symplectic manifold. This is completely new in comparison to submanifolds in positively definite Riemann spaces. So, let $M \subset P$ be a $(2 n-m)$-dimensional submanifold ("constraints") in a symplectic manifold $(P, \gamma)$, e.g., given by equations

$$
F_{a}(\xi)=F_{a}(q, p)=0, \quad a=1, \ldots, m
$$

The system of those functions is functionally independent, at least in some neighbourhood of $M$. Sometimes it is also convenient to take the foliation by submanifolds $M_{a}$

$$
F_{a}(q, p)=c_{a}
$$

where $c_{a}$ are constants. At every $p \in M$ there is a tangent space $T_{p} M$ and its symplectic 
orthogonal (dual) space $T_{p} M^{\perp}$ which consists of vectors $\gamma_{p}$-"orthogonal" to $T_{p} M$ :

$$
T_{p} M^{\perp}=\left\{v \in T_{p} P: \gamma(p)_{a b} v^{b} X^{a} \quad \text { if } \quad X \in T_{p} M\right\}
$$

or in more sophisticated terms: $\left.\langle v\rfloor \gamma_{p}, \cdot\right\rangle \mid T_{p} M=0$. It is a peculiarity of symplectic geometry that $T_{p} M^{\perp}$ need not be complementary to $T_{p} M$. The following class index was introduced to describe this.

If $\operatorname{dim} K_{p}(M)=\operatorname{dim}\left(T_{p} M \cap T_{p} M^{\perp}\right)=k$, then we put $\mathrm{Cl}_{p} M=(k, m-k)$. We are interested mainly in situation when this does not depend on $p$, that is, incidentally, a typical situation. Then we write simply $\mathrm{Cl} M=(k, m-k)$. If $k=m$, then we write simply $\mathrm{Cl} M=\mathrm{I}$ and say that $M$ is co-isotropic. This means that the subspaces $\gamma$-orthogonal to $M$ are tangent to $M$. If $k=0$, then $\mathrm{Cl} M=\mathrm{II}$, and the subspace $\gamma$-orthogonal to $M$ are at the same time transversal (complementary) to $M . \mathrm{Cl} M=\mathrm{I}$ implies that the functions $F_{a}$ in (54) or (55) satisfy respectively $\left\{F_{a}, F_{b}\right\} \mid M=0$ or $\left\{F_{a}, F_{b}\right\}=0$. Similarly, $\mathrm{Cl} M=\mathrm{II}$ implies that $\operatorname{det}\left[\left\{F_{a}, F_{b}\right\}\right] \neq 0$, at least in a neighbourhood of $M$. If $T_{p} M \subset T_{p} M^{\perp}$, then we say that $M$ is isotropic. Then for any pair of tangent vectors at any $p \in M$ we have: $\gamma(p)_{a b} u^{a} v^{b}=0$, when $u, v \in T_{p} M$. If $M$ is isotropic, then $\operatorname{dim} M \leq n$. If $\operatorname{dim} M=n$, i.e., if $T_{p} M=T_{p} M^{\perp}$, we say that $M$ is Lagrangian. It is described by the system of equations $F_{a}=0, a=1, \ldots, n$, $\left\{F_{a}, F_{b}\right\} \mid M=0$, or, if we deal with a foliation by Lagrangian manifolds, i.e., with a polarization, then $F_{a}=c_{a},\left\{F_{a}, F_{b}\right\}=0$. Equations for the Lagrangian submanifold may be solved in the following way with respect to canonical momenta:

$$
p_{i}=\frac{\partial S}{\partial q^{i}}, \quad i=1, \ldots, n,
$$

when it is transversal to the fibres of constant $q^{a}, a=1, \ldots, n$. Let us denote the corresponding manifold by $\mathfrak{m}_{S}$. The Hamilton-Jacobi equation

$$
\Omega\left(\ldots, q^{\mu}, \ldots ; \ldots, \frac{\partial S}{\partial q^{\mu}}, \ldots\right)=0
$$

means that $\mathfrak{m}_{S}$ belongs to the zero-valued surface of $\Omega$. We have used here the Greek symbols $\mu$ to indicate that the time variable may be included into coordinates. For example, in non-relativistic mechanics:

$$
\frac{\partial S}{\partial t}+H\left(t, \ldots, q^{i}, \ldots ; \ldots, \frac{\partial S}{\partial q^{i}}, \ldots\right)=0
$$

The integrability condition for the system of Hamilton-Jacobi equations with functions $\Omega_{v}$, $v=0,1, \ldots, n$, is given by the equation $\left\{\Omega_{\mu}, \Omega_{v}\right\}=0$, i.e., the manifold $\Omega_{\mu}=0, \mu=$ $0,1, \ldots, n$, has the class I, i.e., is co-isotropic. 
One can show that on every regular submanifold $M$ the assignment $M \ni p \mapsto K_{p}(M)=$ $T_{p} M^{\perp} \cap T_{p} M$ is an integrable distribution, therefore, the quotient manifold $P^{\prime}(M)=$ $M / K(M)$ carries the canonical symplectic structure $\gamma^{\prime}$ such that $\gamma \| M=\pi^{*} \gamma^{\prime}$, where $\pi: M \rightarrow P^{\prime}(M)$ is the natural projection. Obviously, $K(M)$ denotes the system of leaves of the distribution. It is clear that $\operatorname{dim} P^{\prime}(M)=2(n-(m+k) / 2)$.

Lagrange manifolds, i.e., isotropic ones of dimension $n$, are placed, as seen from the formula, only on co-isotropic, i.e., first class submanifolds. And if they are transversal to the configuration $Q$-fibres ( $X$-fibres), then using the formula (57) we obtain the Hamilton-Jacobi equations (58), (59) or simply

$$
A\left(\ldots, q^{i}, \ldots, \ldots, \frac{\partial S}{\partial q^{i}}, \ldots\right)=a
$$

for the potential $S$. Every $\mathfrak{m}_{S} \subset M$ is composed of the foliation of singular fibres $K(M)$. Singular fibres, first of all one-dimensional ones, i.e., integral curves of the Hamiltonian vector fields $X_{F}, X_{\Omega}$ are classical trajectories. Those of which $\mathfrak{m}_{S}$ are composed were called by Synge coherent families with the potential $S[18,19]$. As seen, they correspond to quasiclassical wave functions. And classically, being dependent on $n$ parameters, they correspond to the complete integrals of Hamilton-Jacobi equation (59). Let us summarize our symplectic interpretation of them.

In quantum mechanics the eigenstates of the physical quantity represented by the Hermitian operator A are given by density operators $\widehat{\rho}$ satisfying the operator eigenequation $\boldsymbol{\rho}$. Let us stress that in general this is the equation both on $\rho$ and $a$. Taking its Hermitian conjugate we obtain $\boldsymbol{\rho} \mathbf{A}=a \boldsymbol{\rho}$. One can write these equations as $(\mathbf{A}-a \mathrm{Id}) \boldsymbol{\rho}=0, \boldsymbol{\rho}(\mathbf{A}-a \mathrm{Id})=$ 0 . This is the afore-mentioned informative aspect of the eigenequation. But just as it was within the Weyl-Wigner-Moyal-Ville framework, this information context implies the formal consequence, but qualitatively a completely different symmetry property, namely the invariance of $\rho$ under the unitary group generated by $\mathbf{A}:(1 / i \hbar)[\mathbf{A}, \boldsymbol{\rho}]=(1 / i \hbar)(\mathbf{A} \boldsymbol{\rho}-\boldsymbol{\rho} \mathbf{A})=$ 0 . Therefore, in the classical limit one must assume that the quasiclassical $\rho$ satisfies a pair of mathematically independent, but physically interpretable just as above, conditions: $A \rho=a \rho$ — information, $\{A, \rho\}=0$ - symmetry.

Let us introduce the set of operators: $E_{\boldsymbol{\rho}}:=\{\mathbf{F} \in B(H): \mathbf{F} \boldsymbol{\rho}=0\}$. In principle $B(H)$ denotes the set of bounded operators acting in the Hilbert space $H$. Although, to be honest, one can weaken this assumption. It is also clear that similarly as in classical statistics, the following holds in quanta:

$$
-S\left(\rho_{1}\right)=\operatorname{Tr}\left(\rho_{1} \ln \rho_{1}\right) \leq \operatorname{Tr}\left(\rho_{2} \ln \rho_{2}\right)=-S\left(\rho_{2}\right),
$$

when $E_{\boldsymbol{\rho}_{1}} \subset E_{\boldsymbol{\rho}_{2}}$. In other words, the larger $E_{\boldsymbol{\rho}}$, the greater informational content of $\boldsymbol{\rho}$. Of course, we mean here the quantum concept of the Shannon entropy and the mathematical sense of $\operatorname{Tr}(\rho \ln \rho)$. Quantum pure states are defined in such a way that $E_{\rho}$ is a maximal ideal. It answers uniquely the maximal number of experimental questions. There exists then the one-dimensional linear subspace $V \subset H$ such that $E_{\rho}$ consists of operators which vanish 
on $V$,

$$
E_{\rho}=\{\mathbf{F} \in B(H): \mathbf{F} \mid V=0\}
$$

This means that the subspace $V \subset H$ given by $V=\bigcap_{\mathbf{F} \in E_{\rho}} \operatorname{KerF}$ satisfies conditions: $\boldsymbol{\rho}(H)=$ $V, \boldsymbol{\rho} \mid V=\operatorname{Id}_{V}, \boldsymbol{\rho} \rho=\boldsymbol{\rho}$, therefore, $\rho$ is a projector of $H$ onto $V$. The entropy (information) takes on $\rho$ the minimal (maximal) value, $\operatorname{Tr}(\rho \ln \rho)=0$. When $P=T^{*} Q$, then the formulae (47), (48) may be literally applied together with the Bohr-Sommerfeld quantum rules: $\oint \omega=$ $\oint p_{i} d q^{i}=n h$ on any closed curve on $\mathfrak{m}_{S}$. This defines the quantized values of $a^{i}$ in terms of integers and Planck constant.

Expression for the Van Vleck determinant is correct independently on the additional phase space structures like the affine one. Just because of the structure of this determinant. This is seen from the density formula:

$$
\mathcal{V}=\operatorname{det}\left[\frac{\partial S}{\partial q^{i} \partial a^{j}}\right] d q^{1} \wedge \ldots \wedge d q^{n} \otimes d a^{1} \wedge \ldots \wedge d a^{n}
$$

Moreover, it turns out that this determinant is much more general and even the cotangent bundle structure is not necessary for it. Namely, let us assume a pair of polarizations, i.e., a pair of complementary foliations of a general phase space $(P, \gamma)$ by Lagrangian manifolds. Let us observe that $P$ need not be identical with $T^{*} Q$ and everything we assume is just a pair of foliations. Lagrangian submanifolds of any foliation have a local affine structure. Introducing coordinates $q^{i}, a^{i}$, we can formally describe them in terms of equations: $p_{i}=$ $\partial S(q, a) / \partial q^{i}$, but $S$ is non-unique up to the gauging: $S \mapsto S+\varphi \circ \mathrm{pr}_{1}+\Psi \circ \mathrm{pr}_{2}$, where $\mathrm{pr}_{1}$, $\mathrm{pr}_{2}$ are projections from $P$ to the $Q, \mathbb{R}^{n}$-manifolds. But this gauging does not influence the value of the Van Vleck determinant.

It is interesting to see what follows when we consider Hamiltonian and quantum dynamics in a homogeneous formulation of Hamiltonian/quantum dynamics. So, let us consider the motion of a particle in an $(n+1)$-dimensional space-time manifold $X$ and take a complete integral $S\left(x^{\mu}, a^{i}\right)$ depending on $n$ arbitrary constants. Then instead of the above quantities we obtain the following vector-density object:

$$
\mathcal{V}=D^{\mu} d x^{0} \wedge d x^{1} \wedge \ldots \wedge \mu \wedge \ldots \wedge d x^{n} \otimes d a^{1} \wedge \ldots \wedge d a^{n}
$$

where $D^{\mu}$ is a minor of the matrix $\left[\partial^{2} S /\left(\partial x^{\mu} \partial a^{i}\right)\right]$ obtained by removing the $\mu$-th column. The symbol $\mu$ in the exterior product means that $d x^{\mu}$ is omitted. It is clear that the above expressions imply that

$$
\frac{\partial j^{\mu}}{\partial x^{\mu}}=0
$$

where $j^{\mu}=(-1)^{\mu} D^{\mu}$. This formula is geometrically correct, because $j^{\mu}$ is a contravariant vector density of weight one. Therefore, the left-hand side of (65) is well defined in 
spite of using the usual partial differentiation. One can easily check that it follows from (58). In particular, if $\Omega$ in (58) equals the non-relativistic $\Omega=p_{0}+H\left(x^{0}, x^{i} ; p_{i}\right)=$ $-E+H\left(t, q^{i}, p_{i}\right)$ (E denotes the energy variable), then $j^{\mu}$ in (64), (65) equals the formerly written non-relativistic four-current

$$
\left(j^{\mu}\right)=\left(\operatorname{det}\left[\frac{\partial^{2} S}{\partial q^{i} \partial a^{j}}\right], \operatorname{det}\left[\frac{\partial^{2} S}{\partial q^{i} \partial a^{j}}\right] \frac{\partial H}{\partial p_{i}}\left(q, \frac{\partial S}{\partial q}\right)\right) .
$$

This $j^{\mu}$ satisfies the continuity equation (65) in virtue of (40). For the general relativistically written $\Omega\left(x^{\mu}, p_{\mu}\right)$, one obtains the four-current density, e.g., for the quasiclassical Klein-Gordon equation. The current (66) corresponds to some choice of the complete integral of Hamilton-Jacobi equations.

Let us mention that for the system of Hamilton-Jacobi equations

$$
\Omega_{\wedge}\left(x^{\mu}, \frac{\partial S}{\partial x^{\mu}}\right)=0
$$

we obtain generalized continuity equations. However, there is no place to stop at this topic here. In any case $j^{\mu}$ is also built of the complete integral of (67).

It is difficult not to be astonished by the fact that the above structures were not discovered some hundred years earlier. They are based on the purely classical and deeply geometric concepts. As mentioned, this may be explained only by the fact that the Planck constant was not known then. To be more precise, it was hidden deeply in the thickest of radiation theory and its thermodynamics.

Let us mention some additional facts. We said that the Van Vleck symbol may be assigned to any complementary pair of polarizations $Q \times \mathbb{R}^{n} \ni(q, a) \rightarrow V(q, a)$. It may be interpreted in a statistical way due to its structure of the double scalar density. Indeed, the quantity $\mathcal{V}(q, a)=\operatorname{det}\left[\partial^{2} S / \partial q^{i} \partial a^{j}\right]$ may be dualistically interpreted as the density of probability both in $Q$ and in $\mathbb{R}^{n}$. If $A \subset Q, B \subset \mathbb{R}^{n}$, then

$$
P(A, B)=\int_{A \times B} \mathcal{V}(q, a) d q^{1} \ldots d q^{n} d a^{1} \ldots d a^{n}
$$

may be interpreted as the quasiclassical probability that the system with values of integrals of motion in $B \subset \mathbb{R}^{n}$ will be found in the region $A \subset Q$ of the configuration space. And conversely, it is equal to the probability that the system placed in $A \subset Q$ will show the values of integration constants in $B \subset \mathbb{R}^{n}$. To be honest, in general they are non-normalized to unity relative probabilities.

When performing pull-backs of probability densities on $Q$ to $\mathfrak{m}_{S}$, we obtain some probability distributions on the Lagrangian manifold. Therefore, quasiclassical pure quantum states may be interpreted as probability distributions concentrated on submanifolds $\mathfrak{m}_{S}$. So, their supports are $n$-dimensional and distinguished by the fact that $\gamma \| \mathfrak{m}_{S}=0$. Quasiclassical 
mixed states are usually smeared out as $2 n$-dimensional probability distributions on $P=$ $T^{*} Q$.

Let us quote yet some another quasiclassical structures. To do this we begin with the linear symplectic spaces. Let $D(P)$ denote the set of all linear Lagrangian subspaces of $P$. Let $M \subset$ $P$ be some co-isotropic linear subspace of $P$ and $D(M) \subset D(P)$ denote the set Lagrangian subspaces contained in $M$. One can show that any $\mathfrak{m} \subset D(P)$ intersects $M$ along some at least $(n-m)$-dimensional isotropic subspace. But the singular fibre of $M$, i.e., $M^{\perp} \subset M$ is $m$-dimensional. Therefore, the subspace

$$
E_{M}(\mathfrak{m}):=\mathfrak{m} \cap M+M^{\perp}
$$

is also Lagrangian and contained in $M$. Therefore, without any additional structure $M$ gives rise to the mapping $E_{M}: D(P) \rightarrow D(M)$ with the following properties:

1. $E_{M}$ is a retraction onto the subset $D(M)$, moreover, it is a projection:

$$
E_{M} \mid D(M)=\operatorname{id}_{D(M)}, \quad E_{M} \circ E_{M}=E_{M} .
$$

2. $M, N$ are co-isotropic and compatible, i.e., $M \cap N$ is also co-isotropic, then $E_{M}, E_{N}$ commute and

$$
E_{M} \circ E_{N}=E_{N} \circ E_{M}=E_{M \cap N} .
$$

3. If $E_{M} \circ E_{N}=E_{N} \circ E_{M}$, then $M, N$ are compatible and (71) holds.

4. If $f: P \rightarrow P$ is a symplectic ( $\gamma$-preserving) mapping, then

$$
E_{f(M)}=F \circ E_{M} \circ F^{-1},
$$

where $F: D(P) \rightarrow D(P)$ is induced by $f$.

This is interesting and easily interpretable in terms of quasiclassical wave functions. The relationship with the corresponding quantum relations is also readable. Let us illustrate this with the following simple example.

We consider an affine (or linear) phase space with affine coordinates $\left(q^{i}, p_{i}\right)$. Then we have $\omega=p_{i} d q^{i}, \gamma=d \omega=d p_{i} \wedge d q^{i}$. Let us take as $M$ the manifold of states on which the momentum variable $p_{1}$ takes on a fixed values $b$,

$$
M=\left\{p \in P: p_{1}(p)=b\right\}, \quad D(P) \ni \mathfrak{m}=\left\{p \in P: q^{i}(p)=a^{i}\right\}
$$

where $i=1, \ldots, n$, therefore, $M$ is a manifold with fixed values of $p_{1}$ equal to $b$, and $\mathfrak{m}$ is the Lagrangian space (the carrier of a quasiclassical state) with fixed positions $a^{i}$. One can easily 
show that

$$
E_{M}(\mathfrak{m})=\left\{p \in P: p_{1}(p)=b, q^{2}(p)=a^{2}, \ldots, q^{n}(p)=a^{n}\right\}
$$

Therefore, if we fix the value of $p_{1}$ with the help of $E_{M}$, we result in a complete indeterminacy of $q^{1}$. But this is just the classical uncertainty principle. Simply on the classical, or rather semi-classical level, it is not a point in the phase space, but rather Legendre submanifold, or to be more precise, a probability distribution on it, that is a counterpart of the quantum wave state.

$P$ was assumed here to be a linear space endowed with a symplectic structure. But it turns out that the above prescription may be globalized to the general symplectic manifold. Roughly speaking, this follows from its "flatness" which makes it similar to a linear symplectic space in finite domains due to the existence of Darboux coordinates which enable one to write $\gamma=d p_{i} \wedge d q^{i}$. This holds in every symplectic manifold, not necessarily cotangent bundle, locally, but in finite domains. Indeed, let $M \subset P$ be a co-isotropic submanifold, $K(M)-$ its singular foliation, and $\mathfrak{m} \subset P-$ Lagrangian submanifold. The manifolds $M$ and $\mathfrak{m}$ need not to intersect; in such situation we say that $E_{M}(\mathfrak{m})=\varnothing$. In this way the empty set $\varnothing$ is joined to $D(P)$. It corresponds to the vanishing wave function. We put also $E_{M}(\varnothing)=$ $\varnothing$. Similarly we do when $\mathfrak{m}, M$ intersect in a non-clean way, i.e., otherwise than linear subspaces. Let us assume the generic case, when $\mathfrak{m}, M$ intersect in a regular way, i.e., when $T_{p} \mathfrak{m} \cap T_{p} M=T_{p}(\mathfrak{m} \cap M)$ for any $p \in \mathfrak{m} \cap M$. To be more precise, we assume that the subset of points $p$ at which this is satisfied is a Lagrangian submanifold. Obviously, $\mathfrak{m} \cap$ $M$ is an isotropic submanifold. If the intersection $\mathfrak{m} \cap M$ is regular at its every point $p$, then $E_{M}(\mathfrak{m})$ is defined as the maximal extension of $\mathfrak{m} \cap M$ by the singular foliation $K(M)$, $E_{M}(\mathfrak{m})=\pi^{-1}(\mathfrak{m} \cap M)$. It is evidently Lagrangian and $E_{M}: D(P) \rightarrow D(M)$ satisfies the above properties (70)-(72). One should mention only that (72) is satisfied by every canonical mapping, i.e., every diffeomorphism preserving the two-form $\gamma$. The finite-dimensional symplectic group is replaced by the infinite-dimensional group "parameterized" by arbitrary functions.

It is also interesting to know that there are classical counterparts of superpositions and scalar products. We have seen that pure states are represented in a sense by probability distributions concentrated on Lagrange manifolds $\mathfrak{m}_{S}$. But the function $S$ itself, i.e., the phase of wave functions, is not contained in the corresponding analogy. One should adjoint an additional dimension, action, and consider locally the manifolds $Q \times \mathbb{R}, P \times \mathbb{R}$, or rather $Q \times \mathrm{SU}(1)$, $P \times \mathrm{SU}(1)$. To be mathematically more honest, one should use the principal fibre bundles with the bases $Q, P$ and the structure group $\mathbb{R}_{\text {additive }}$ or $\mathrm{SU}(1)$ multiplicative. The corresponding geometry of the contact fibre bundle $C$ over $P$ is locally given by

$$
\Omega=p_{i} d q^{i}-d z
$$

Take the set of Legendre submanifolds corresponding to the complete integral $\left\{S_{a}: a \in A\right\}$ of the Hamilton-Jacobi equation (40), (58), (59). These solutions may be represented by their 
diagrams (locally) in $Q \times \mathbb{R}$ or $Q \times \mathrm{SU}(1)$ :

$$
\text { Graph } S_{a}=\left\{\left(q, S_{a}(q)\right): q \in Q\right\}
$$

The independence of the Hamilton-Jacobi equation on the algebraic presence of the variable $S$ implies that for any value of $a$ the function $S_{a}+t(a)$ with $t(a) \in \mathbb{R}$ is a solution too. The Hamilton-Jacobi equation imposes only conditions on the tangent elements of functions, therefore, the envelope of diagrams $\left\{\left(q, S_{a}+t(a)\right): q \in Q\right\}$ denoted by

$$
\operatorname{Env}_{a \in A}\left\{\left(q, S_{a}(q)+t(a)\right): q \in Q\right\}
$$

also represents some solution of (40), (58), (59). The arbitrariness of these solutions corresponds exactly to the arbitrariness of functions $t: A \rightarrow \mathbb{R}$. Let us repeat that (77) is a diagram of the set of values $\{(q, S(q)): q \in Q\}$, where the function $S$ is obtained from the family of $S_{a}$-s and $t$ in the following way:

i We start from equations:

$$
\frac{\partial}{\partial a^{i}}\left(S_{a}(q)+t(a)\right)=0
$$

and solve them, at least in principle, with respect to $a$. One obtains (also in principle) some $q$-dependent solution, $a(q)$.

ii This solution is substituted to $S_{a}(q)+t(a)$ and one obtains the expression denoted by the Stat-symbol,

$$
S(q)=S_{a(q)}(q)+t(a(q))=\operatorname{Stat}_{a \in A}\left(S_{a}(q)+t(a)\right) .
$$

This follows from the theory of the Hamilton-Jacobi equation. But the same may be shown from "deriving" the continuous superpositions of wave functions satisfying the Schrödinger equation, by performing the WKB-limit transition $\hbar \rightarrow 0$ in the following expression:

$$
\int \sqrt{w(a)} \exp \left(\frac{i}{\hbar} t(a)\right) \sqrt{D(a)} \exp \left(\frac{i}{\hbar} S(q, a)\right) d_{n} a
$$

In the WKB-limit $\hbar \rightarrow 0$ one obtains just (79) as the limit condition. Let us mention that for any function $S$ on a differentiable manifold $A$ the symbol Stat $f$ denotes the value of $S$ at the stationary point $a \in A$, where $d S_{a}=0$. If there are many stationary points, then $S$ in (79) is multivalued. This means that the quasiclassical superposition consists of several waves with various values of phases. We omit the relatively complicated quasiclassical behaviour of moduli, just for simplicity. Similarly, for the pair of wave functions $\Psi_{1}=\sqrt{D_{1}} \exp (i / \hbar) S_{1}$, $\Psi_{2}=\sqrt{D_{2}} \exp (i / \hbar) S_{2}$ we can investigate the quasiclassical behaviour of the scalar product:

$$
\left\langle\Psi_{1} \mid \Psi_{2}\right\rangle=\int \Psi_{1}(q) \Psi_{2}(q) d_{n} q=\int \sqrt{D_{1} D_{2}} \exp \left(\frac{i}{\hbar}\left(S_{1}-S_{2}\right)\right) d_{n} q
$$


Denoting $\left\langle\Psi_{1} \mid \Psi_{2}\right\rangle=\sqrt{D} \exp (i / \hbar) S$ and applying the method of stationary phase we again obtain $S=\operatorname{Stat}\left(S_{2}-S_{1}\right)=$ Stat $_{q \in Q}\left(S_{2}(q)-S_{1}(q)\right)$, where just as previously, Stat $_{q \in Q} \varphi(q)$ denotes the value of $\varphi$ at the point $q \in Q$, where the differential of $\varphi$ vanishes:

$$
d \varphi_{q}=0
$$

The situation is simple when (82) has exactly one solution. If there are a few $q_{1}, \ldots, q_{k}$, then the scalar product is a superposition of ones with the corresponding phases

$$
\lambda_{1} \exp \left(\frac{i}{\hbar} \varphi_{1}\right)+\ldots+\lambda_{k} \exp \left(\frac{i}{\hbar} \varphi_{k}\right)
$$

where $\lambda$-s are obtained from the quasiclassical limits of $D$. In any case there is a multivalued phase $\varphi_{1}, \ldots, \varphi_{k}$. If (82) has no solutions, then we say that $\Psi_{1}, \Psi_{2}$ are quasiclassically orthogonal.

It would be nice to express those concepts in terms of the contact geometry (75). Let us remind that the Lagrange submanifolds $\mathfrak{m}_{S} \subset P$ represent rather the density operators of quasiclassical pure states than their wave functions. The latter ones are represented by horizontal lifts of Lagrange submanifolds, i.e., by the maximal, thus $n$-dimensional horizontal submanifolds in $C$, i.e., such ones $\mathfrak{M}$ that $\Omega \| \mathfrak{M}=0$. In particular, they are given as $\mathfrak{M}_{S}=$ hor $\mathfrak{m}_{S}$, where

$$
\mathfrak{M}_{S}:=\left\{\left(d S_{q}, S(q)\right): q \in Q\right\}
$$

But they need not be so; another extreme example is the horizontal lift of $T_{q}^{*} Q$,

$$
\mathfrak{M}_{q}:=\left(T_{q}^{*} Q, 0\right)=\left\{(p, 0): p \in T_{q}^{*} Q\right\}
$$

Intermediate examples between (84), (85) are labelled by pairs $(M, S)$ where $M \subset Q$ is a submanifold of $Q$ and $S: M \rightarrow \mathbb{R}$ is a real-valued function on $M$. The corresponding Legendre submanifold in $T^{*} Q$ is given by

$$
\mathfrak{M}_{(M, S)}:=\left\{(p, S(\pi(p))): \pi(p) \in M, p \mid T_{\pi(p)} M=d S_{\pi(p)}\right\}
$$

$\pi: T^{*} Q \rightarrow Q$ is the natural projection of the co-tangent bundle onto its base.

Let us now translate the above formulae into the language of contact geometry. Some similarities to the rigorous quantum expressions will be obvious. The vertical fibre bundle action of the structural group $\mathbb{R}$ or $\mathrm{SU}(1)$ when operating on the elements $\mathfrak{M}$ of the set of Legendre manifolds $\mathcal{H}(C)$ will be denoted as follows: $[t] \mathfrak{M}:=\left\{g_{t}(z): z \in \mathfrak{M}\right\}$, where $g_{t}$ is 
the action of the group element in $C$. If $T$ is a countable subset of the group elements, then we put:

$$
T \mathfrak{M}:=\bigcup_{t \in G}[t] \mathfrak{M}=\bigcup_{t \in G}\left\{g_{t}(z): z \in \mathfrak{M}\right\} .
$$

In the case of empty sets (which correspond formally to zero), we have $\varnothing \mathfrak{M}=\varnothing, T \varnothing=\varnothing$.

Now let us express the quasiclassical "superposition" and phases of the "scalar products" in terms of the contact geometry. Let $M \subset C$ be a submanifold. Its characteristic subset $\Sigma(M)$ is defined as the set of all points $z \in M$ at which $\Omega_{z} \mid T_{z} M=0$. In practical applications we often deal with the situation that $\Sigma(M)$, which is always horizontal, is at the same time $n$-dimensional, therefore, it is a Legendre submanifold, i.e., an element of $\mathcal{H}(C)$. Let us assume that $\left\{\mathfrak{M}_{a}: a \in A\right\}$ is a family of elements of $\mathcal{H}(C)$, i.e., a family of Legendre submanifolds. We say that its superposition is the maximal element of $\mathcal{H}(C)$ which is contained in the determinant set of $\bigcup_{a \in A} \mathfrak{M}_{a}$. We denote it by $\mathfrak{M}=E_{a \in A} \mathfrak{M}_{a}$. Without going into details we show with the help of examples below that this superposition is in fact, from the point of view of $Q \times \mathbb{R}$, the envelope or "generalized envelope" of the family of surfaces:

i Let us again consider the contact manifold $C=T^{*} Q \times \mathbb{R}$ with the natural contact form $p_{i} d q^{i}-d z$. We take a manifold $A$ parameterizing functions $S_{a}(q)=S(q, a)$ and the coefficients function $f: A \rightarrow \mathbb{R}$. $S$ gives rise to the following family of Legendre manifolds:

$$
\mathfrak{M}_{a}:=\mathfrak{M}_{S(\cdot, a)}=\left\{\left(d S(\cdot, a)_{q}, S(q, a)\right): q \in Q\right\} .
$$

If it happens (it need not be so) that

$$
E_{a \in A}[f(a)] \mathfrak{M}_{a}=\mathfrak{M}_{S}=\left\{\left(d S_{q}, S(q)\right): q \in Q\right\}
$$

then we obtain $S(q)=\operatorname{Stat}_{a \in A}(S(q, a)+f(a))$. And this means that the manifold $\xi_{S}:=$ $\{(q, S(q)): q \in Q\} \subset Q \times \mathbb{R}$ is really the literal envelope of the family of submanifolds $\xi_{a}:=\xi_{S(\cdot, a)}=\{(q, S(q, a)): q \in Q\} \subset Q \times \mathbb{R}$.

ii Again we consider $C=T^{*} Q \times \mathbb{R}$ with the following natural contact form: $\Omega=p_{i} d q^{i}-$ $d z$. And we take again the $q$-localized Legendre manifold $\mathfrak{M}_{q}=\left(T_{q}^{*} Q, 0\right)$, and $\mathfrak{M}_{S}=$ $\left\{\left(d S_{q}, S(q)\right): q \in Q\right\}$. One can show that $\mathfrak{M}_{S}=E_{q \in Q}[S(q)] \mathfrak{M}_{q}$; this is a kind of irregular envelope from the point of view of the geometry of $Q$.

iii We take a linear space $V$ as a manifold $Q$. Then we have $T^{*} Q \simeq V \times V^{*}$ and $C \simeq V \times V^{*} \times$ $\mathbb{R}$. And then we take as Legendre submanifolds the following ones with well-defined positions, $\mathfrak{M}[x]=\left\{(x, p, 0): p \in V^{*}\right\}$, and with the fixed canonical momenta, $\mathfrak{M}[p]=$ $\{(x, p,\langle p, x\rangle): x \in V\}$. One can easily check the next rules of the quasi-classical Fourier analysis: $\mathfrak{M}[p]=E_{x \in V}[\langle p, x\rangle] \mathfrak{M}[x], \mathfrak{M}[x]=E_{p \in V^{*}}[-\langle p, x\rangle] \mathfrak{M}[p]$. And then for any "phase" function $S: V \rightarrow \mathbb{R}$ we have $\mathfrak{M}_{S}=E_{x \in V}[S(x)] \mathfrak{M}[x]=E_{p \in V^{*}}[\widehat{S}(p)] \mathfrak{M}[p]$ with the following translation rules between $S$ and $\widehat{S}$ : 


$$
\widehat{S}(p)=\operatorname{Stat}_{x \in V}(S(x)-\langle p, x\rangle), S(x)=\operatorname{Stat}_{p \in V^{*}}(\widehat{S}(p)+\langle p, x\rangle) .
$$

When we take into account that the analogy between phases of $\Psi(x)$ and $\widehat{\Psi}(p)$ is seriously accepted, we see immediately the obvious quasiclassical relationship between $\Psi(x)$ and $\widehat{\Psi}(p)$. It is based on the concept of generalized envelope.

Let us also notice that all those concepts are invariant with respect to the special contact transformations in $C$. First of all, let us remind that $u: C \rightarrow C$ is a special contact transformation when it preserves $\Omega, u^{*} \Omega=\Omega$. If $u$ is such and $U: \mathcal{H}(C) \rightarrow \mathcal{H}(C)$ is the corresponding transformation of $\mathcal{H}(C)$, then $U E_{a \in A}\left[t_{a}\right] \mathfrak{M}_{a}=E_{a \in A}\left[t_{a}\right] U \mathfrak{M}_{a}$.

Now let us begin with the concept of the vertical distance, i.e., scalar product of Legendre submanifolds. Let us take a pair of Legendre manifolds $\mathfrak{M}_{1}, \mathfrak{M}_{2} \in \mathcal{H}(C)$ with the property that their Lagrange projections $\mathfrak{m}_{1}, \mathfrak{m}_{2}$ and also $\mathfrak{m}_{1} \cap \mathfrak{m}_{2}$ are connected and simply-connected. Then there exists exactly one number $t \in \mathbb{R}$ (or $\exp (i t) \in \mathrm{SU}(1)$ ) of the property that $\left(g_{t} \mathfrak{M}_{1}\right) \cap \mathfrak{M}_{2} \neq \varnothing$. This number $t$ or better its unitary exponent $\exp (i t)$ is the scalar product of $\mathfrak{M}_{1}$ and $\mathfrak{M}_{2}$. To be more precise, the classical scalar product is $\exp (i t)$, where $t$ is its phase. More generally, we say that the vertical distance, or the Huygens scalar product $\left[\mathfrak{M}_{1} \mid \mathfrak{M}_{2}\right]$ of the pair of Legendre submanifolds $\mathfrak{M}_{1}, \mathfrak{M}_{2}$ is a subset of $\mathbb{R}$ (or exponentially of SU(1)) such that if $t \in\left[\mathfrak{M}_{1} \mid \mathfrak{M}_{2}\right]$, then $\mathfrak{M}_{2} \cap g_{t}\left(\mathfrak{M}_{1}\right) \neq \varnothing$. If $\left[\mathfrak{M}_{1} \mid \mathfrak{M}_{2}\right]$ is empty, then we say that $\mathfrak{M}_{1}, \mathfrak{M}_{2}$ are orthogonal. Their Lagrange projections $\mathfrak{m}_{1}, \mathfrak{m}_{2}$ onto $P=T^{*} Q$ are then disjoint. It is clear that any mapping $U: \mathcal{H}(C) \rightarrow \mathcal{H}(C)$ generated by a special contact transformation $u: C \rightarrow C$ is then "unitary" in the sense of the scalar product $[\cdot, \cdot]$, i.e., $\left[U \mathfrak{M}_{1} \mid U \mathfrak{M}_{2}\right]=\left[\mathfrak{M}_{1} \mid \mathfrak{M}_{2}\right]$ for any pair of Legendre submanifolds $\mathfrak{M}_{1}, \mathfrak{M}_{2}$.

Let $M \subset P=T^{*} Q$ be any co-isotropic submanifold and $\mathcal{H}_{M}(C) \subset \mathcal{H}(C)$ denote the set of Legendre submanifolds with Lagrange projections to $P=T^{*} Q$ placed within $M$. Then the operations $E_{M}$ on Lagrangian submanifolds introduced above may be canonically lifted to the operations $\Pi_{M}$ acting on the horizontal lifts of $\mathfrak{m} \subset D(P)=D\left(T^{*} Q\right)$. Namely, for any co-isotropic $M$ there exists the canonical mapping $\Pi_{M}: \mathcal{H}(C) \rightarrow \mathcal{H}_{M}(C)$ with the property: $\Pi \circ \Pi_{M}=\Lambda_{M} \circ \Pi,\left(\Pi_{M} \mathfrak{M}\right) \cap \mathfrak{M}=\left(\pi^{-1}(M)\right) \cap \mathfrak{M}$, where $\Pi: \mathcal{H}(C) \rightarrow D(P)=D\left(T^{*} Q\right)$ is the natural projection induced by the fibre bundle projection $\pi: C \rightarrow P$. In fact, $\Pi_{M} \mathfrak{M}$ is the horizontal lift of $\Lambda_{M} \mathfrak{m}$ which contains $\mathfrak{M} \cap\left(\pi^{-1}(M)\right)$. If $\mathfrak{m}$ intersects $M$ in a regular way, then $\Pi_{M} \mathfrak{M}$ is a maximal extension of the intersection $\mathfrak{M} \cap\left(\pi^{-1}(M)\right)$ along the fibres of the $\Omega$-horizontal lift $K^{\Omega}(M)=$ lift $K(M)$. If $\mathfrak{m} \cap M=\varnothing$ or if it is not regular, then it is assumed that $\Pi_{M} \mathfrak{M}=\varnothing$.

Let us repeat again that $\Pi_{M}$ satisfy the properties (69)-(72) modified by the admitted empty-set values: $\Pi_{M} \mid \mathcal{H}_{M}(C)=\operatorname{Id}_{\mathcal{H}_{M}}(C), \Pi_{M} \circ \Pi_{M}=\Pi_{M}, \Pi_{M} \circ \Pi_{N}=\Pi_{N} \circ \Pi_{M}=\Pi_{M \cap N}$ if $\mathrm{Cl} M \cap N=$ I. If $\Pi_{M} \circ \Pi_{N}=\Pi_{N} \circ \Pi_{M}$, then $M \cap N$-compatible and the both sides equal $\Pi_{M \cap N}$. For any special contact transformation $\Pi_{f M}=F \circ \Pi_{M} \circ F^{-1}$, where $F$ is a transformation of $\mathcal{H}(C)$ induced by $f$.

It is clear that every special contact transformation $u$, i.e., diffeomorphisms of $C$ preserving $\Omega$, projects to $P$ onto canonical transformation $\underline{u}$ preserving $\gamma, \pi \circ u=\underline{u} \circ \pi$. Let $\left\{\mathfrak{M}_{q}: q \in Q\right\}$ be a system of Legendre submanifolds of $C$ such that $\bigcup_{q \in Q} \mathfrak{M}_{q}$ is an image of a cross-section of $C$ over $P$, such that the projections to $P, \mathfrak{m}_{q}$ form a polarization, i.e., a family of mutually 
disjoint Lagrange submanifolds of $P$. Then $u$ acts on $\mathfrak{M}_{q}$ in such a way that

$$
U \mathfrak{M}_{q}=E_{q^{\prime} \in Q} U\left(q^{\prime}, q\right) \mathfrak{M}_{q^{\prime}}, \quad U\left(q^{\prime}, q\right)=\left[\mathfrak{M}_{q^{\prime}} \mid U \mathfrak{M}_{q}\right]
$$

Then for any superposition-envelope $\mathfrak{M}=E_{q \in Q}[S(q)] \mathfrak{M}_{q}$ the following holds:

$$
U \mathfrak{M}=E_{q \in Q}\left[S^{\prime}(q)\right] \mathfrak{M}_{q}=E_{q \in Q}[S(q)] U \mathfrak{M}_{q}
$$

where $S^{\prime}(q)=\operatorname{Stat}_{q^{\prime} \in Q}\left(U\left(q, q^{\prime}\right)+S\left(q^{\prime}\right)\right)$. This is an obvious analogue and the phase classical limit of the linear rule for superposition of wave functions with the phase factors $\exp (i / \hbar) S\left(q^{\prime}\right)$. And $U\left(q, q^{\prime}\right)$ is just the generating function of the type $W(q, Q)=U\left(q, q^{\prime}\right)$. And a similar construction may be built for other types of generating functions.

Let us also mention that a similar "quasilinear" representation may be achieved for other operations on Legendre submanifolds, not necessarily ones induced by diffeomorphisms acting in $C$. For example, let us consider $\Pi_{M}$, i.e., $\mathfrak{M}=E_{q \in Q}[S(q)] \mathfrak{M}_{q}, \Pi_{M} \mathfrak{M}=$ $E_{q \in Q}\left[S^{\prime}(q)\right] \mathfrak{M}_{q}$. Then we have $S^{\prime}(q)=\operatorname{Stat}_{q^{\prime} \in Q}\left(S\left(q^{\prime}\right)+\Pi_{M}\left(q^{\prime}, q\right)\right)$, where $\Pi_{M}: Q \times Q \rightarrow$ $\mathbb{R}$ is the Legendre propagator $\Pi_{M}\left(q^{\prime}, q\right)=\left[\mathfrak{M}_{q^{\prime}} \mid \Pi_{M} \mathfrak{M}_{q}\right]$. This is again the envelope-like Huygens-quasilinear rule. Using the Stat-symbol one can also write a nice-looking analogue of the Feynman-Stückelberg "sum over paths" rule.

Incidentally, let us remind that by the $W$-type generating function $W\left(q, q^{\prime}\right)$ we mean such one that the corresponding canonical transformation $(q, p) \mapsto\left(q^{\prime}, p^{\prime}\right)$ is given by $p_{i}=\partial W\left(q, q^{\prime}\right) / \partial q^{i}, p_{i}^{\prime}=-\partial W\left(q, q^{\prime}\right) / \partial q^{\prime i}$. Not every canonical transformation does possess such a function in the usual sense, but it may be replaced by a more general generating function. There is no place here to get deeper into details, cf. e.g. [11].

Let us also stress a few other facts connected with the notion of (generalized) envelope. Consider the compatible system of Hamilton-Jacobi equations: $F_{a}\left(\ldots, x^{\mu}, \ldots ; \ldots, \partial S / \partial x^{\mu}, \ldots\right)=0$. Any fibre of the cotangent bundle may be $\Lambda_{M}$-projected onto $D(M)$ - the set of Lagrange submanifolds of $M, \mathfrak{m}_{x}:=\Lambda_{M}\left(T_{x}^{*} X\right)$. Then, every $(n+1)$-dimensional $\pi^{-1}\left(\mathfrak{m}_{X}\right)$ (where $\left.n=\operatorname{dim} X\right)$ is foliated by the family of Legendre lifts of $\mathfrak{m}_{X}$. When $C=T^{*} X \times \mathbb{R}$ or $C=T^{*} X \times \mathrm{U}(1)$ those lifts are $\mathfrak{M}_{(x, c)}:=\Pi_{M}\left(T_{x}^{*} X, c\right)$. Then locally

$$
\mathfrak{m}_{x} \cap T_{y}^{*} X=\left\{d \sigma(x, \cdot)_{y}\right\}, \quad \mathfrak{M}_{x} \cap\left(T_{y}^{*} X \times \mathbb{R}\right)=\left\{d \sigma(x, \cdot)_{y}, \sigma(x, y)\right\} .
$$

For every pair of points $x, y \in X$ we define the quantity $\sigma_{M}(x, y)$, namely

$$
\sigma_{M}(x, y)=\int_{l(x, y)} \omega=\int_{\ell(x, y)} p_{\mu} d x^{\mu}
$$

where $\ell(x, y)$ is any curve placed on a singular fibre containing $x, y \in X$. This $\sigma_{M}$ is a fundamental solution, $\Pi_{M} \mathfrak{M}_{S}=\mathfrak{M}_{S^{\prime}}, \Lambda_{M} \mathfrak{m}_{S}=\mathfrak{m}_{S^{\prime}}$, where $S^{\prime}(x)=\operatorname{Stat}_{y}\left(S(y)+\sigma_{M}(y, x)\right)$. When $\Sigma \subset X$ is a Cauchy surface for our Hamilton-Jacobi system, then $S(x)=$ 
Stat $_{q \in \Sigma}\left(f(q)+\sigma_{M}(q, x)\right)$, where $f: \Sigma \rightarrow \mathbb{R}$ are initial data. Therefore, the two-point characteristic function is a Hamilton-Jacobi propagator. The idempotence property of $\Lambda_{M}$, $\Pi_{M}$ implies that $\sigma_{M}(x, y)=\operatorname{Stat}_{z}\left(\sigma_{M}(x, z)+\sigma_{M}(z, y)\right)$. Let us quote an interesting example of the free material point in Galilean space-time. Then

$$
\frac{1}{\hbar} \sigma_{M}(x, y)=\frac{1}{\hbar} S(a, z ; q, t)=\frac{m}{2 \hbar(t-z)} g_{i j}\left(q^{i}-a^{i}\right)\left(q^{j}-a^{j}\right)
$$

When the Van Vleck determinant $\operatorname{det}\left[\partial^{2} S / \partial q^{i} \partial a^{j}\right]$ is multiplied by some normalization constant, then the Van Vleck solution

$$
\sqrt{\operatorname{det}\left[\frac{\partial^{2} S}{\partial q^{i} \partial a^{j}}\right]} \exp \left(\frac{i m}{2 \hbar(t-z)} g_{k l}\left(q^{k}-a^{k}\right)\left(q^{l}-a^{l}\right)\right)
$$

becomes

$$
\mathcal{K}(\bar{\zeta}, \tau)=\left(\frac{m}{2 \pi i \hbar \tau}\right)^{n / 2} \exp \left(\frac{i m}{2 \hbar \tau} \bar{\zeta}^{2}\right)
$$

where $\tau=t-z, \xi^{k}=q^{k}-a^{k}$, and $\bar{\xi}^{2}=g_{k l} \xi^{k} \xi^{l}$. The normalization we have accepted is given by $\lim _{\tau \rightarrow 0} \mathcal{K}(\bar{\zeta}, \tau)=\delta(\bar{\zeta})$, where $\mathcal{K}$ is the usual rigorous quantum propagator for the Schrödinger equations:

$$
i \hbar \frac{\partial \Psi}{\partial t}=-\frac{\hbar^{2}}{2 m} \Delta \Psi=-\frac{\hbar^{2}}{2 m} g^{i j} \partial_{i} \partial_{j} \Psi,
$$

in spite of the fact that it was obtained in a purely classical way.

Summary of Section 3: In this section we have reminded some classical problems concerning classification of submanifolds in the classical phase space. Their classical interpretation in terms of symplectic and contact structures was discussed. Again it turns out that the classical limits are Huygens constructions based on the envelope concepts. The quantum and classical relationships between information and symmetry were discussed. This analysis shows again, without any use of the Weyl-Wigner-Moyal-Ville product that it is probability distributions concentrated on $n$-dimensional Lagrange manifolds that corresponds to the quantum pure states on the classical level. The homogeneous Van Vleck objects corresponding to the evolution problems were discussed. In particular, this may be used to the analysis of the Klein-Gordon equation. Discussed is the WKB-limit of certain quantum expressions like superpositions of wave functions and their scalar products. Again one obtains expressions based on the envelope concepts and the Huygens-like operations on the set of Lagrangian manifolds. Classical counterparts of the projection operators were found. The envelopes of diagrams of phases of wave functions are geometrically interpreted in terms of contact geometry. This is a geometric picture valid for all types of the eikonal equations. It enables one to interpret also the purely classical concepts like generating functions of canonical transformations in quantum-like form based on the envelopes of diagrams of phases. Roughly speaking, the envelope represents the phase of the classical superposition. It is shown that the quantum propagation for the free evolution Schrödinger equation may be smoothly guessed on the purely classical level, in terms of the Van Vleck determinant. 


\section{Nonlinearity program in quantum mechanics}

Let us consider a finite-level quantum mechanical system. We try to interpret the Schrödinger equation as a usual self-adjoint equation of mathematical physics, just as if it was to be a classical one. If both the first- and second-order time derivatives of the state vector $\Psi$ are to be admitted, the Lagrange function may be postulated as

$$
L(1,2)=i \alpha \Gamma_{\bar{a} b}\left(\bar{\Psi}^{\bar{a}} \dot{\Psi}^{b}-\dot{\Psi}^{\bar{a}} \Psi^{b}\right)+\beta \Gamma_{\bar{a} b} \dot{\Psi}^{\bar{\Psi}^{a}} \dot{\Psi}^{b}-\gamma_{\Gamma} H_{\bar{a} b} \bar{\Psi}^{\bar{a}} \Psi^{b}
$$

where $\alpha, \beta, \gamma$ are constants and $\Gamma_{\bar{a} b}$ are components of the scalar product. ${ }_{\Gamma} H_{\bar{a} b}$ are components of the Hamiltonian matrix in the covariant form,

$$
{ }_{\Gamma} H_{\bar{a} b}=\Gamma_{\bar{a} c} H_{b}^{c}
$$

whereas $H_{b}^{c}$ are usual mixed tensor components. To be honest, in physics it is this mixed Hamilton operator that is considered as a primary quantity. From the Lagrangian point of view it is a twice covariant form that is primary. Because of this we decided to assume $\mathrm{H}^{c}{ }_{b}$ as a primary quantity, but the Hermitian matrix $H_{\bar{a} b}$ is assumed as the constitutive element in (99). When we are going to introduce a direct nonlinearity to the treatment, we introduce in addition a real-valued potential $V(\Psi, \bar{\Psi})$, e.g.,

$$
V(\Psi, \bar{\Psi})=f\left(\Gamma_{\bar{a} b} \bar{\Psi}^{\bar{a}} \Psi^{b}\right)
$$

where $f$ is a real-valued function of the one real variable.

For the Lagrangian $L=L(1,2)-V$ we obtain the following "Schrödinger", or rather "Schrödinger-Klein-Gordon", equation:

$$
2 i \alpha \frac{d \Psi^{a}}{d t}-\beta \frac{d^{2} \Psi^{a}}{d t^{2}}=\gamma H_{b}^{a} \Psi^{b}+f^{\prime} \Psi^{a}
$$

The comparison with the usual Schrödinger equation tells us that $\alpha=\hbar / 2, \gamma=1$. The energy function for $L=L(1,2)-V$ is given by $\mathcal{E}=\beta \Gamma_{\bar{a} b} \dot{\Psi}^{\bar{a}} \dot{\Psi} b+\gamma_{\Gamma} H_{\bar{a} b} \bar{\Psi}^{\bar{a}} \Psi^{b}+V(\Psi, \bar{\Psi})$. Legendre transformation tells us that the corresponding Hamiltonian in the sense of analytical mechanics is:

$$
\mathcal{H}=\frac{1}{\beta}\left[\Gamma^{a \bar{b}} \pi_{a} \bar{\pi}_{\bar{b}}+i \alpha\left(\pi_{a} \Psi^{a}-\bar{\pi}_{\bar{a}} \bar{\Psi}^{\bar{a}}\right)\right]+\left[\frac{\alpha^{2}}{\beta} \Gamma_{\bar{a} b}+\gamma_{\Gamma} H_{\bar{a} b}\right] \bar{\Psi}^{\bar{a}} \Psi^{b}+V(\Psi, \bar{\Psi}),
$$

where $\pi_{a}, \bar{\pi}_{\bar{a}}$ are canonical momenta conjugate to $\Psi^{a}, \bar{\Psi}^{\bar{a}}$. It is clear that $\mathcal{E}$ is always defined all over the state space. Unlike this, $\mathcal{H}$ is defined all over the phase space only when $\beta \neq 0$. If $\beta=0$, it is defined only on the constraints submanifold. 
The possible nonlinearity of quantum mechanics is due to the term $V(\Psi, \bar{\Psi})$. As mentioned, this is a rather artificial, perturbative nonlinearity. It is definitely better to use the essential nonlinearity of the geometric, group-theoretic origin. To achieve this, one should follow the idea of transition from special to general relativity. The simplest way is to "de-absolutize" the scalar product. Namely, instead of being fixed once for all, the scalar product will be reinterpreted as a dynamical variable. It is to be self-interacting and free of any fixed absolute background. Therefore, in the finite-level theory, its dynamics will be $\operatorname{GL}(n, \mathbb{C})$-invariant. The only natural Lagrangian will follow the structure of affinely-invariant kinetic energy of affinely-rigid body. So, for the metric $\Gamma_{\bar{a} b}$ we postulate the following Lagrangian:

$$
T=L[\Gamma]=\frac{A}{2} \Gamma^{b \bar{c}} \Gamma^{d \bar{a}} \dot{\Gamma}_{\bar{a} b} \dot{\Gamma}_{\bar{c} d}+\frac{B}{2} \Gamma^{b \bar{a}} \Gamma^{d \bar{c}} \dot{\Gamma}_{\bar{a} b} \dot{\Gamma}_{\bar{c} d}
$$

This is the only possibility which is not based on anything absolutely fixed. Obviously, the main term is the first one, controlled by $A$. The $B$-term is a correction, not very essential, but acceptable from the point of view of the assumed $\mathrm{GL}(n, \mathbb{C})$-symmetry.

In the Lagrangian (100), (101) for the wave function the scalar product is also replaced by this new, dynamical version. Due to the resulting very essential nonlinearity following from (104) the quantum-classical gap in a sense becomes diffused. One can hope that in the resulting theory the decoherence phenomena may be explained. For example, if for simplicity we fix $\Psi^{a}$ as constant (non-excited), then we can show that differential equations for $\Gamma$ obtained from (104) have the following solutions: $\Gamma_{\bar{r} s}=G_{\bar{r} s} \exp (E t)^{z}{ }_{s}=\exp (F t)_{\bar{r}}{ }_{\bar{z}} G_{\bar{z} s}$, where $G=\Gamma(0)$ is Hermitian and the forms ${ }_{G} E_{\bar{r} s}=G_{\bar{r} z} E_{s}^{z},\left(F_{G}\right)_{\bar{r} s}=F_{\bar{r}}^{\bar{z}} G_{\bar{z} s}$ are also Hermitian. Let us observe that depending on the initial data $G, E, F$ the scenarios of the evolution of $t \mapsto \Gamma(t)$ may be quite different: oscillatory, exponentially increasing, exponentially decaying. This may suggest that in the rigorous total solutions for $t \mapsto(\Psi(t), \Gamma(t))$ also various phenomena may be predicted, e.g., oscillations, but perhaps also decoherence.

The maximal class of $\operatorname{GL}(n, \mathbb{C})$-invariant Lagrangians $L[\Psi, \Gamma]$ is relatively wide. It seems however that the simplest and at the same time most realistic subclass is given by the following expression:

$$
\begin{aligned}
L & =i \alpha_{1} \Gamma_{\bar{a} b}\left(\bar{\Psi}^{\bar{a}} \dot{\Psi}^{b}-\dot{\Psi}^{\bar{a}} \Psi^{b}\right)+\alpha_{2} \Gamma_{\bar{a} b} \dot{\Psi}^{\bar{\Psi}^{a}} \dot{\Psi}^{b}+\left(\alpha_{3} \Gamma_{\bar{a} b}+\alpha_{4} H_{\bar{a} b}\right) \bar{\Psi}^{\bar{a}} \Psi^{b} \\
& +\alpha_{5} \Gamma^{d \bar{a}} \Gamma^{b \bar{c}} \dot{\Gamma}_{\bar{a} b} \dot{\Gamma}_{\bar{c} d}+\alpha_{6} \Gamma^{b \bar{a}} \Gamma^{d \bar{c}} \dot{\Gamma}_{\bar{a} b} \dot{\Gamma}_{\bar{c} d}-\mathcal{V}(\Psi, \bar{\Psi} ; \Gamma) .
\end{aligned}
$$

The quantities $\alpha_{1}, \ldots, \alpha_{6}$ are real constants. They control all the effects mentioned above. The separation of the $\alpha_{3}$ - and $\alpha_{4}$-terms may look artificial. In fact, their superposition is as a matter of fact one term. Nevertheless, it seems reasonable to separate the true Hamiltonian effect from that following from the identity operator type. As mentioned formerly, to obtain the correct Schrödinger behaviour in the $\Psi$-sector we must put $\alpha_{1}=\hbar / 2, \alpha_{4}=-1$. But of course if $\alpha_{2} \neq 0$, then in addition to the Schrödinger behaviour we have also as usual in analytical mechanics, the acceleration term in $\Psi$. The scheme is a bit obscured because of our dealing with a finite-level system. The extension to the usual quantum mechanics, say in $\mathbb{R}^{3}$, is possible. Besides, let us remind our papers devoted to the study of the $\mathrm{SU}(2,2)$-gauge 
gravitation theory $[12,13]$. There the problem of nonlinearity and the interplay between firstand second-order differential equations for the matter fields appear in a much more evident way.

We do not quote "Schrödinger equation" for the pairs $(\Psi, \Gamma)$ ruled by Lagrangians (105). Their structure is very readable, nevertheless, their strong nonlinearity prevented us from finding their convincing full solutions, when the mutual interaction between $\Psi$ - and $\Gamma$-degrees of freedom is taken into account.

Summary of Section 4: This section was one of the main parts of our study. We are aware that in spite of all similarities and analogies there is still some really quantum kernel of the theory which seems to be incompatible with any attempts of formulating the peaceful coexistence of the unitary "between measurement" and the "reduction-like" phenomena in quantum physics. As usual, the idea of nonlinearity in quantum physics turns out to be attractive. It seems to be the only way to coordinate the "between measurements" unitary evolution and the measurement reduction process. There were various more or less happy ways to introduce nonlinearity; some of them were rather artificial. Our idea resembles the transition from the special to general relativity. Namely, we give up the concept of scalar product fixed once for all and instead consider the scheme in which the wave function and scalar product are both dynamical objects in the mutual interaction. Lagrangian for the scalar product is geometric, invariant under the full linear group and so is the total Lagrangian for the system: wave function and the scalar product. The resulting scheme is nonlinear in an essential, non-perturbative way. There are some indications that the resulting nonlinear system of equations may describe both the "between measurements" evolution and the reduction of state process.

\section{Modifications of the WWMV approach}

We have seen that the mentioned approach was a very fruitful tool for studying the quasi-classical problems and the relationship between information and symmetry. Unfortunately, its literal version applies rigorously only to systems with affine geometry of the classical phase space. There are various ways to generalize those methods, usually based on group theory and deformation techniques. Some of those methods are applicable also to discrete structures.

Let $G$ be a locally compact topological group. Its Haar measure element will be denoted by $d g$. To be honest, to avoid problems with the convergence of integrals, we may assume $G$ to be compact. Let us introduce the following non-local product of functions on $G$ :

$$
(A \perp B)(g)=\int \mathcal{K}\left(g ; g_{1}, g_{2}\right) A\left(g_{1}\right) B\left(g_{2}\right) d g_{1} d g_{2} .
$$

To be honest, we think about the multiplication rule for functions not necessarily on $G$ itself, but rather on its affine space, i.e., on the set on which $G$ acts with trivial isotropy groups. Therefore, we assume the translational invariance, so that $\mathcal{K}\left(g ; g_{1}, g_{2}\right) \equiv \mathcal{K}\left(g_{1} g^{-1}, g_{2} g^{-1}\right)$. The simultaneous assumption of associativity, $A \perp(B \perp C)=(A \perp B) \perp C$ implies that $\mathcal{K}$ must satisfy the following functional equation: 


$$
\int \mathcal{K}\left(g_{1}, g\right) \mathcal{K}\left(g_{2} g^{-1}, g_{3} g^{-1}\right) d g=\int \mathcal{K}\left(g_{1} g^{-1}, g_{2} g^{-1}\right) \mathcal{K}\left(g, g_{3}\right) d g .
$$

When $G$ is a locally compact Abelian group, one can try to translate (107) into the language of Fourier transforms. This is suggested by the convolution-like structure of this condition. Let us remind that the dual group $\widehat{G}$ is the multiplicative group of all continuous homomorphisms of $G$ into $\mathbb{T}=\mathrm{SU}(1)$ - the group of complex numbers of modulus 1 . The Fourier transform of a complex function $\Psi$ on $G$ is the function $\widehat{\Psi}$ on $\widehat{G}$ given by:

$$
\widehat{\Psi}(\chi)=\int \overline{\langle\chi \mid g\rangle} \Psi(g) d g
$$

where $\langle\chi \mid g\rangle$ denotes the value of $\chi \in \widehat{G}$ at $g \in G$. And conversely,

$$
\Psi(g)=\int\langle\chi \mid g\rangle \widehat{\Psi}(\chi) d \chi
$$

Performing the two-argument Fourier transformation on the equation (107) we obtain the following condition:

$$
\widehat{\mathcal{K}}\left(\chi_{1}, \chi_{2} \chi_{3}\right) \widehat{\mathcal{K}}\left(\chi_{2}, \chi_{3}\right)=\widehat{\mathcal{K}}\left(\chi_{1}, \chi_{2}\right) \widehat{\mathcal{K}}\left(\chi_{1} \chi_{2}, \chi_{3}\right)
$$

This is an equation for the factor of ray representations. It is clear that the Fourier representation of (106) in the Abelian case is given by:

$$
(\widehat{A} \top \widehat{B})(\chi)=\int \widehat{\mathcal{K}}\left(\chi_{1}, \chi_{1}^{-1} \chi\right) \widehat{A}\left(\chi_{1}\right) \widehat{B}\left(\chi_{1}^{-1} \chi\right) d \chi_{1}
$$

This is the $\widehat{K}$-twisted convolution of functions. It becomes the usual convolution when $\widehat{K} \equiv 1$. In analogy to (111) one defines the twisted convolution of measures.

A similar operation, i.e., twisted convolution of functions, or more generally, one of measures, may be defined in any locally compact topological group,

$$
(A \top B)(g)=\int \omega\left(h, h^{-1} g\right) A(h) B\left(h^{-1} g\right) d h,
$$

again in the sense of Haar measure $d h$. This product is associative for any group $G$, not necessarily the Abelian one, if and only if the mentioned functional equation (110) holds, i.e., if $\omega$ behaves like the factor of the ray representation, $\omega\left(g_{1}, g_{2}\right) \omega\left(g_{1} g_{2}, g_{3}\right)=$ $\omega\left(g_{1}, g_{2} g_{3}\right) \omega\left(g_{2}, g_{3}\right)$. One can show that there is a relationship between the twisted convolutions of functions (or measures) over $G$ and the usual ones in some $G$-extension of the circle group $\mathbb{T}=S U(1)$. The choice of the phase-space group $G$ depends on the particular model. 
Let us go back to the situation when $G$ is the group which models the configuration space, not the phase space. And we assume $G$ to be Abelian. The phase space $\mathcal{G}$ will be given by $\mathcal{G}=G \times \widehat{G}$, where the dual group $\widehat{G}$ is to model the "space of momenta". In analogy to the natural symplectic two-form on the linear space $V \times V^{*}$ we introduce the following two-character on $\mathcal{G}, \zeta: \mathcal{G} \times \mathcal{G} \rightarrow \mathbb{C}$ (the two-character, because $\zeta(\xi, \cdot), \zeta(\cdot, \xi)$ are characters on $\mathcal{G}$ for any $\xi \in \mathcal{G})$ :

$$
\zeta\left(\left(x_{1}, \pi_{1}\right),\left(x_{2}, \pi_{2}\right)\right)=\left\langle\pi_{1} \mid x_{2}\right\rangle \overline{\left\langle\pi_{2} \mid x_{1}\right\rangle}=\frac{\left\langle\pi_{1} \mid x_{2}\right\rangle}{\left\langle\pi_{2} \mid x_{1}\right\rangle}
$$

It is non-singular in the sense that the mappings $\xi \mapsto \zeta(\xi, \cdot), \xi \mapsto \zeta(\cdot, \xi)$ are isomorphisms of $\mathcal{G}$ onto $\mathcal{G}$. Wave functions in the position and momentum representations are defined as amplitudes on $G$ and $\widehat{G}$ respectively. The group actions of $G, \widehat{G}$ on wave functions are given by the following unitary representations:

$$
(U(x) \Psi)(y)=\Psi\left(x^{-1} y\right), \quad(V(\pi) \Psi)(y)=\langle\pi \mid y\rangle \Psi(y)
$$

The second operator is obviously equal to the argument translation when the momentum representation is used: $(V(\pi) \Psi)^{\wedge}(\lambda)=\widehat{\Psi}\left(\pi^{-1} \lambda\right)$. One can check easily that the following fundamental commutation relation is satisfied:

$$
U(x) V(\pi) U(x)^{-1} V(\pi)^{-1}=\overline{\langle\pi \mid x\rangle}=\langle\pi \mid x\rangle^{-1} .
$$

Following the ideas of the Weyl prescription we define the following unitary operators: $W_{p}(x, \pi)=\langle\pi \mid x\rangle^{p} U(x) V(\pi)=\langle\pi \mid x\rangle^{p-1} V(\pi) U(x)$. If in $G$ or $\widehat{G}$ there exists a unique square root like in $\mathbb{R}^{n}$, then we put $p=1 / 2$ and then $W\left(x^{-1}, \pi^{-1}\right)=W(x, \pi)^{-1}$. But in general it does not exist and we retain $p$ as a non-defined label. We take the linear closure:

$$
\mathbf{A}=\int \widehat{A}(x, \pi) \mathbf{W}_{p}(x, \pi) d x d \pi
$$

$\widehat{A}$ denoting the Fourier transform of $A$. The corresponding "multiplication" rule for the functions $A, B$ is based on the kernel:

$$
K_{p}\left(\left(x_{1}, \pi_{1}\right),\left(x_{2}, \pi_{2}\right)\right)=\int\left\langle\pi_{1} \mid \xi\right\rangle\left\langle\eta \mid x_{1}\right\rangle\left\langle\pi_{2} \mid \zeta\right\rangle\left\langle\theta \mid x_{2}\right\rangle\langle\eta \mid \zeta\rangle^{1-p}\langle\theta \mid \xi\rangle^{-p} d \xi d \eta d \zeta d \theta
$$

One can ask about the analogue of the "continuous canonical basis" of the usual $H^{+}$-algebra over $\mathbb{R}^{2 n}$ :

$$
\begin{aligned}
& \rho_{\bar{q}_{1}, \bar{q}_{2}}(\bar{q}, \bar{p})=\delta\left(\bar{q}-\frac{1}{2}\left(\bar{q}_{1}+\bar{q}_{2}\right)\right) \exp \left(\frac{i}{\hbar} \bar{p} \cdot\left(\bar{q}_{2}-\bar{q}_{1}\right)\right) \\
& \rho_{\bar{p}_{1}, \bar{p}_{2}}(\bar{q}, \bar{p})=\delta\left(\bar{p}-\frac{1}{2}\left(\bar{p}_{1}+\bar{p}_{2}\right)\right) \exp \left(\frac{i}{\hbar}\left(\bar{p}_{1}-\bar{p}_{2}\right) \cdot \bar{q}\right) .
\end{aligned}
$$


Those bases satisfied:

$$
\begin{aligned}
q^{i} * \rho_{\bar{q}_{1}, \bar{q}_{2}}=q_{1}{ }^{i} \rho_{\bar{q}_{1}, \bar{q}_{2},} & \rho_{\bar{q}_{1}, \bar{q}_{2}} * q^{i}=q_{2}{ }^{i} \rho_{\bar{q}_{1}, \bar{q}_{2},} \\
p_{i} * \rho_{\bar{p}_{1}, \bar{p}_{2}}=p_{1 i} \rho_{\bar{p}_{1}, \bar{p}_{2},}, & \rho_{\bar{p}_{1}, \bar{p}_{2}} * p_{i}=p_{2 i} \rho_{\bar{p}_{1}, \bar{p}_{2}} .
\end{aligned}
$$

It turns out, however, that when there is no square-rooting in $G, \widehat{G}$, there are some problems. Namely, in $\mathbb{R}^{2 n}$ we could use both $\zeta$ and $\zeta^{2}$ as kernels. But in a general Abelian group it is essential that we use $\zeta$, not $\zeta^{2}$ as a kernel. The analogue of (120), (121) reads: $A * \rho_{x, y}=$ $A(x) \rho_{x, y}, \rho_{x, y} * A=\rho_{x, y} A(y)$. We obtain $\rho_{x_{1}, x_{2}}(x, \pi)=\delta\left(x_{1} x_{2} x^{-1}\right)\left\langle\pi \mid x_{1} x^{-1}\right\rangle$. If $x_{1} x_{2}$ fails to be a square, then $g_{x_{1} x_{2}}=0$. It is not yet clear for us if our procedure was improper or if we deal with the real superselection rule.

Let us observe that the group commutator does not feel the choice of $p$ :

$$
W_{p}\left(x_{1}, \pi_{1}\right) W_{p}\left(x_{2}, \pi_{2}\right) W_{p}\left(x_{1}, \pi_{1}\right)^{-1} W_{p}\left(x_{2}, \pi_{2}\right)^{-1}=\zeta\left(\left(x_{1}, \pi_{1}\right),\left(x_{2}, \pi_{2}\right)\right) \mathrm{Id} .
$$

If $G=\mathbb{Z}^{n}$ or $\mathbb{T}^{n}=(\mathrm{SU}(1))^{n}$, then the mentioned problem with $\zeta$ may be connected with what in solid state physics is known as so-called Umklapp-Prozessen.

Let us observe also that in a sense one can use the following kernel of the non-local product of functions over the discrete group $\mathbb{Z}^{2 n}: K(\bar{n}, \bar{m})=\exp \left(i B_{a b} n^{a} m^{b}\right)$, where $\left[B_{a b}\right]$ is the real skew-symmetric matrix. Nevertheless, the resulting product will have then some strange features.

Let us finish with some remarks concerning the asymptotics of "large quantum numbers" in the quasi-classical limit transition. For simplicity we consider only the wave functions of the planar rotators, $\Psi_{n}(\varphi)=\exp (\operatorname{in} \varphi), n \in \mathbb{Z}$, where $\varphi$ is the angular variable. $\Psi_{n}$ is proportional to the eigenfunction of the angular momentum with the eigenvalue $n \hbar$,

$$
\frac{\hbar}{i} \frac{\partial}{\partial \varphi} \Psi_{n}=\hbar n \Psi_{n}
$$

Clearly

$$
\Psi_{n} \simeq \exp \left(\frac{i}{\hbar}(n \hbar) \varphi\right) ;
$$

it is just $l_{n}=n \hbar$ that is interpreted as the physical value of the angular momentum. But the analysis $\hbar \rightarrow 0$ does not work directly. We must take superpositions of quickly-oscillating eigenequations,

$$
\Psi(\varphi)=\sum_{n} c_{n} \exp (\operatorname{in} \varphi),
$$

where the sequence $\mathbb{Z} \ni n \mapsto c_{n} \in \mathbb{C}$ is concentrated in a range

$$
n_{0}-\Delta n \ll n \ll n_{0}+\Delta n
$$


It is assumed here that $n_{0} \gg \Delta n \gg 1$ and the sequence is assumed to be slowly-varying in the range (126), so that

$$
\frac{\left|c_{n+1}-c_{n}\right|}{\left|c_{n}\right|} \ll 1
$$

It follows from the Fourier theory that approximately (125) may be replaced by:

$$
\Psi(\varphi)=\int c(k) \exp (i k \varphi) d k,
$$

where at the discrete values of $k=n c(k)$ equals $c_{n}$ and changes slowly, e.g., linearly between them. Then $\Psi(\varphi)$ is well concentrated and one can consider it as a quickly-vanishing at infinity function on $\mathbb{R}$. And then one substitutes $k=p / \hbar$ and further on the previous asymptotics $\hbar \rightarrow 0$ may be used. The same when there are more degrees of freedom. Let us remind that it was just this limit transition we have used in the theory of angular momentum [14-16]. By the way, the conditions (126), (127) enabled one to remove artificial picks of the basic wave functions at $k=2 \pi$ in SU(2). Namely, the subsequent picks have opposite signs and mutually cancel when (126), (127) are satisfied.

Summary of Section 5: We have mentioned here about some generalizations of the Weyl-Wigner-Moyal-Ville procedure. They are based on some group-theoretic models and may be perhaps helpful in the formally "classical", although in fact quantum, approach to dynamics.

\section{Conclusions}

We have discussed certain problems concerning the relationship between classical and quantum theories. Analyzed are both differences and formal similarities between them. What concerns similarities, we show that in contrast to some popular views, it is not points in the classical phase space but rather $n$-dimensional Lagrangian submanifolds in the phase space that corresponds to the quantum pure states. More precisely, the classical "pure state" is a probability distribution on the Lagrange manifold, or rather on its horizontal Legendre lift to the contact space. Here $n$ is the number of degrees of freedom and the contact space is, roughly speaking, the Cartesian product of the phase space by $\mathbb{R}$ or $U(1)$ with geometry given by $p_{i} d q^{i}-d z$. It was shown that superpositions, scalar products, etc. are defined in the set of Legendre manifolds and have some formal properties of the corresponding quantum concepts. They are based on the Huygens notion of envelope of the wave fronts. This was shown both directly on the basis of limit transition in the Weyl-Wigner-Moyal-Ville formalism and on the basis of general symplectic language. Nevertheless, it is clear that quantum mechanics with its reduction and decoherence problems is something completely different than the classical theory. We try to show that unlike this view, there is a nonlinear modification of quantum theory which perhaps would be free of the mentioned paradoxes. It is based on the classical language of variational principles and on the concept of dynamical scalar product. The system consisting of wave function and scalar product satisfies an essentially nonlinear, non-perturbative dynamical equation. Its characteristic nonlinearity seems to be able to describe analytically the decoherence process. Finally, we review some 
generalization of the Weyl-Wigner-Moyal-Ville formalism and discuss the quasi-classical limit in terms of "large quantum numbers".

The general conclusion/hypothesis is that perhaps there is no such a gap between classics and quanta as one commonly believes.

\section{Acknowledgements}

This paper partially contains results obtained within the framework of the research project N N501 049540 financed from the Scientific Research Support Fund in the years 2011-2014. The authors are greatly indebted to the Polish Ministry of Science and Higher Education for this financial support.

\section{Author details}

J. J. Sławianowski* and V. Kovalchuk*

Institute of Fundamental Technological Research, Polish Academy of Sciences, Warsaw, Poland

*Address all correspondence to: jslawian@ippt.pan.pl, vkoval@ippt.pan.pl

\section{References}

[1] Abraham R., Marsden JE. Foundations of Mechanics (second edition). London-Amsterdam-Don Mills-Ontario-Sydney-Tokyo: The Benjamin-Cummings Publishing Company, Inc.; 1978.

[2] Arnold VI. Mathematical Methods of Classical Mechanics (second edition). Graduate Texts in Mathematics 60, New York-Berlin-Heidelberg-London-Paris-Tokyo-Hong Kong-Barcelona-Budapest: Springer-Verlag; 1989.

[3] Caratheodory C. Variationsrechnung und Partielle Differential-Gleidungen Erster Ordnung. Leipzig: B.G. Teubner; 1956.

[4] Doebner HD., Goldin GA. Introducing Nonlinear Gauge Transformations in a Family of Nonlinear Schrödinger Equations. Phys. Rew. A 1996;54 3764-3771.

[5] Doebner HD., Goldin GA., Nattermann P. Gauge Transformations in Quantum Mechanics and the Unification of Nonlinear Schrödinger Equations. J. Math. Phys. 1999;40 49-63; quant-ph:9709036.

[6] Landau LD., Lifshitz EM. Quantum Mechanics. London: Pergamon Press; 1958.

[7] Mackey GW. The Mathematical Foundations of Quantum Mechanics. New York: The Benjamin-Cummings Publishing Company, Inc.; 1963.

[8] Moyal JE. Quantum Mechanics as a Statistical Theory. Proc. Cambridge Philosophical Society 1949;45 99-124. 
[9] Moyal JE. Stochastic Processes and Statistical Physics. Journal of the Royal Society B $1949 ; 11$ 150-210.

[10] Sławianowski JJ. Uncertainty, Correspondence and Quasiclassical Compatibility. In: Uncertainty Principle and Foundations of Quantum Mechanics. London-New York-Sydney-Toronto: John Wiley \& Sons; 1977.

[11] Sławianowski JJ. Geometry of Phase Spaces. Warsaw/Chichester-New York-Brisbane-Toronto-Singapore: PWN - Polish Scientific Publishers/John Wiley \& Sons; 1991.

[12] Sławianowski JJ. Order of Time Derivatives in Quantum-Mechanical Equations. In: Pahlavani MP. (ed.). Measurements in Quantum Mechanics. Rijeka: InTech; 2012. p57-74.

[13] Sławianowski JJ., Kovalchuk V. Schrödinger and Related Equations as Hamiltonian Systems, Manifolds of Second-Order Tensors and New Ideas of Nonlinearity in Quantum Mechanics. Rep. Math. Phys. 2010;65(1) 29-76.

[14] Sławianowski JJ., Kovalchuk V., Martens A., Gołubowska B., Rożko EE. Quasiclassical and quantum systems of angular momentum. Part I. Group algebras as a framework for quantum-mechanical models with symmetries. Journal of Geometry and Symmetry in Physics 2011;21 61-94.

[15] Sławianowski JJ., Kovalchuk V., Martens A., Gołubowska B., Rożko EE. Quasiclassical and quantum systems of angular momentum. Part II. Quantum mechanics on Lie groups and methods of group algebras. Journal of Geometry and Symmetry in Physics 2011;22 67-94.

[16] Sławianowski JJ., Kovalchuk V., Martens A., Gołubowska B., Rożko EE. Quasiclassical and quantum systems of angular momentum. Part III. Group algebra su(2), quantum angular momentum and quasiclassical asymptotics. Journal of Geometry and Symmetry in Physics 2011;23 59-95.

[17] Svetlichny G. Nonlinear Quantum Mechanics on the Planck Scale. Int. J. Theor. Phys. 2005;44(11) 2051-2058.

[18] Synge JL. Geometrical Mechanics and de Broglie Waves. Cambridge: Cambridge University Press; 1954.

[19] Synge JL. Classical Dynamics. Berlin: Springer; 1960.

[20] Van Vleck JH. The Correspondence Principle in the Statistical Interpretation of Quantum Mechanics. Proc. Nat. Acad. Sci. USA 1928;14(2) 178-188. 
